\title{
Nobel lecture: When atoms behave as waves: Bose-Einstein condensation and the atom laser*
}

\author{
Wolfgang Ketterle ${ }^{\dagger}$ \\ Department of Physics, MIT-Harvard Center for Ultracold Atoms, \\ and Research Laboratory of Electronics, Massachusetts Institute of Technology, \\ Cambridge, Massachusetts 02139
}

(Published 20 November 2002)

\section{INTRODUCTION}

The lure of lower temperatures has attracted physicists for the past century, and with each advance towards absolute zero, new and rich physics has emerged. Laypeople may wonder why "freezing cold" is not cold enough. But imagine how many aspects of nature we would miss if we lived on the surface of the sun. Without inventing refrigerators, we would only know gaseous matter and never observe liquids or solids, and miss the beauty of snowflakes. Cooling to normal earthly temperatures reveals these dramatically different states of matter, but this is only the beginning: many more states appear with further cooling. The approach into the kelvin range was rewarded with the discovery of superconductivity in 1911 and of superfluidity in helium-4 in 1938. Cooling into the millikelvin regime revealed the superfluidity of helium-3 in 1972. The advent of laser cooling in the 1980s opened up a new approach to ultralow-temperature physics. Microkelvin samples of dilute atom clouds were generated and used for precision measurements and studies of ultracold collisions. Nanokelvin temperatures were necessary to explore quantum-degenerate gases, such as Bose-Einstein condensates first realized in 1995. Each of these achievements in cooling has been a major advance, and recognized with a Nobel prize.

This paper describes the discovery and study of BoseEinstein condensates (BEC's) in atomic gases from my personal perspective. Since 1995, this field has grown explosively, drawing researchers from the communities of atomic physics, quantum optics, and condensedmatter physics. The trapped ultracold vapor has emerged as a new quantum system that is unique in the precision and flexibility with which it can be controlled and manipulated. At least 30 groups have now created condensates, and the publication rate on Bose-Einstein condensation has soared following the discovery of the gaseous condensates in 1995 (see Fig. 1).

\footnotetext{
*The 2001 Nobel Prize in Physics was shared by E. A. Cornell, Wolfgang Ketterle, and E. Wieman. This lecture is the text of Professor Ketterle's address on the occasion of the award. The lecture of Professors Cornell and Wieman is reprinted in the July 2002 issue of Reviews of Modern Physics.

${ }^{\dagger}$ URL: http://cua.mit.edu/ketterle_group/
}

The phenomenon of Bose-Einstein condensation was predicted long ago, in a 1925 paper by Albert Einstein (Einstein, 1925b) using a method introduced by Satyendra Nath Bose to derive the black-body spectrum (Bose, 1924). When a gas of bosonic atoms is cooled below a critical temperature $T_{c}$, a large fraction of the atoms condenses in the lowest quantum state. Atoms at temperature $T$ and with mass $m$ can be regarded as quantum-mechanical wave packets that have a spatial extent on the order of a thermal de Broglie wavelength $\lambda_{d B}=\left(2 \pi \hbar^{2} / m k_{B} T\right)^{1 / 2}$. The value of $\lambda_{d B}$ is the position uncertainty associated with the thermal momentum distribution and increases with decreasing temperature. When atoms are cooled to the point where $\lambda_{d B}$ is comparable to the interatomic separation, the atomic wave packets "overlap" and the gas starts to become a "quantum soup" of indistinguishable particles. Bosonic atoms undergo a quantum-mechanical phase transition and form a Bose-Einstein condensate (Fig. 2), a cloud of atoms all occupying the same quantum-mechanical state at a precise temperature (which, for an ideal gas, is related to the peak atomic density $n$ by $n \lambda_{d B}^{3}=2.612$ ). If the atoms are fermions, cooling gradually brings the gas closer to being a "Fermi sea" in which exactly one atom occupies each low-energy state.

Creating a BEC is thus simple in principle: make a gas extremely cold until the atomic wave packets start to overlap! However, in most cases quantum degeneracy would simply be preempted by the more familiar transitions to a liquid or solid. This more conventional condensation into a liquid and solid can only be avoided at extremely low densities, about a hundred-thousandth

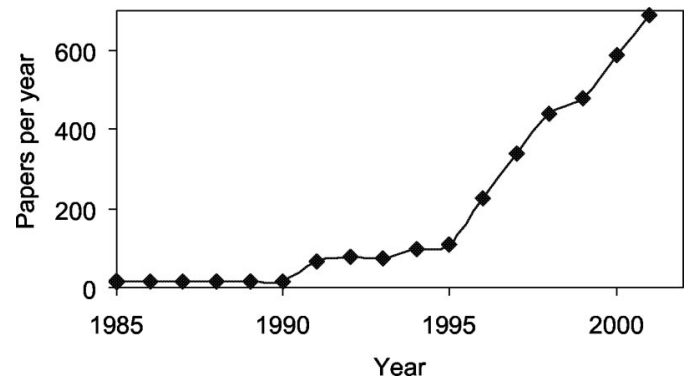

FIG. 1. Annual number of published papers which have the words "Bose" and "Einstein" in their title, abstracts, or keywords. The data were obtained by searching the ISI (Institute for Scientific Information) database. 


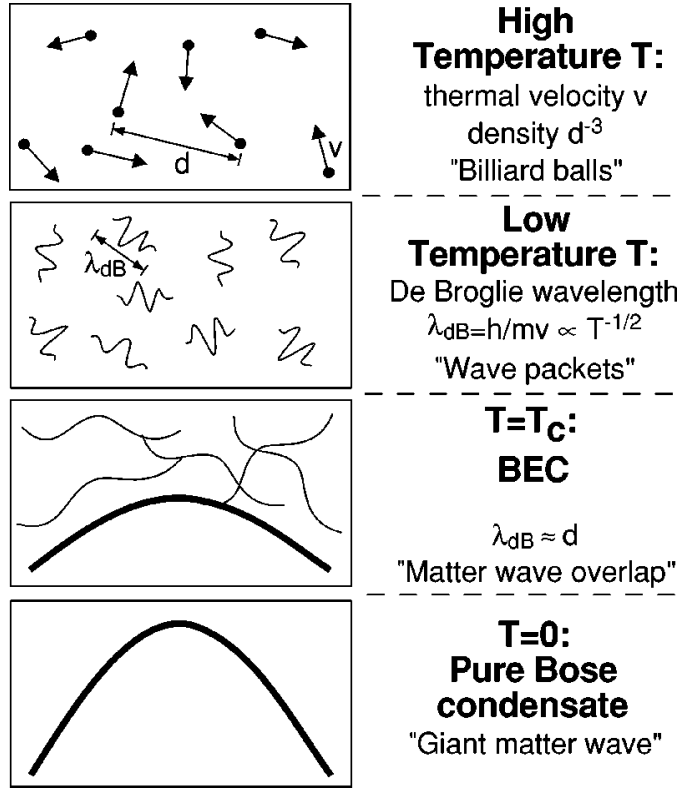

FIG. 2. Criterion for Bose-Einstein condensation. At high temperatures, a weakly interacting gas can be treated as a system of "billiard balls." In a simplified quantum description, the atoms can be regarded as wave packets with an extension of their de Broglie wavelength $\lambda_{d B}$. At the BEC transition temperature, $\lambda_{d B}$ becomes comparable to the distance between atoms, and a Bose condensate forms. As the temperature approaches zero, the thermal cloud disappears, leaving a pure Bose condensate.

the density of normal air. Under those conditions, the formation time of molecules or clusters by three-body collisions (which is proportional to the inverse density squared) is stretched to seconds or minutes. Since the rate of binary elastic collisions drops only proportional to the density, these collisions are much more frequent. Therefore thermal equilibrium of the translational degree of freedom of the atomic gas is reached much faster than chemical equilibrium, and quantum degeneracy can be achieved in an effectively metastable gas phase. However, such ultralow density lowers the temperature requirement for quantum degeneracy into the nanokelvin to microkelvin range.

The achievement of Bose-Einstein condensation required first the identification of an atomic system which would stay gaseous all the way to the BEC transition, and second, the development of cooling and trapping techniques to reach the required regime of temperature and density. Even around 1990, it was not certain that Nature would provide us with such a system. Indeed, many people doubted that BEC could ever be achieved, and it was regarded as an elusive goal. Many believed that pursuing BEC would result in new and interesting physics, but whenever one would come close, some new phenomenon or technical limitation would show up. A news article in 1994 quoted Steve Chu: "I am betting on Nature to hide Bose condensation from us. The last 15 years she's been doing a great job" (Taubes, 1994).

In brief, the conditions for BEC in alkali gases are reached by combining two cooling methods. Laser cool- ing is used to precool the gas. The principle of laser cooling is that scattered photons are on average blueshifted with respect to the incident laser beam. As a result, the scattered light carries away more energy than has been absorbed by the atoms, resulting in net cooling. Blueshifts are caused by Doppler shifts or ac Stark shifts. The different laser cooling schemes are described in the 1997 Nobel lectures in physics (Chu, 1998; CohenTannoudji, 1998; Phillips, 1998). After the precooling, the atoms are cold enough to be confined in a magnetic trap. Wall-free confinement is necessary, otherwise the atoms would stick to the surface of the container. It is noteworthy that similar magnetic confinement is also used for plasmas which are too hot for any material container. After magnetically trapping the atoms, forced evaporative cooling is applied as the second cooling stage (Masuhara et al., 1988; Ketterle and van Druten, 1996; Walraven, 1996). In this scheme, the trap depth is reduced, allowing the most energetic atoms to escape while the remainder rethermalize at steadily lower temperatures. Most BEC experiments reach quantum degeneracy between $500 \mathrm{nK}$ and $2 \mu \mathrm{K}$, at densities between $10^{14}$ and $10^{15} \mathrm{~cm}^{-3}$. The largest condensates are of 100 million atoms for sodium, and a billion for hydrogen; the smallest are just a few hundred atoms. Depending on the magnetic trap, the shape of the condensate is either approximately round, with a diameter of 10-50 $\mu \mathrm{m}$, or cigar-shaped with about $15 \mu \mathrm{m}$ in diameter and $300 \mu \mathrm{m}$ in length. The full cooling cycle that produces a condensate may take from a few seconds to as long as several minutes.

After this short overview, I want to provide the historical context for the search for BEC and then describe the developments which led to the observation of BEC in sodium at MIT. Finally, some examples will illustrate the novel physics which has been explored using BoseEinstein condensates. A more detailed account of the work of my group has been presented in four comprehensive review papers (Ketterle and van Druten, 1996; Ketterle et al., 1999; Ketterle and Inouye, 2001; Stamper-Kurn and Ketterle, 2001).

\section{BEC AND CONDENSED-MATTER PHYSICS}

Bose-Einstein condensation is one of the most intriguing phenomena predicted by quantum statistical mechanics. The history of the theory of BEC is very interesting, and is nicely described in the biographies of Einstein (Pais, 1982) and London (Gavroglu, 1995) and reviewed by Griffin (1999). For instance, Einstein made his predictions before quantum theory had been fully developed, and before the differences between bosons and fermions had been revealed (Einstein, 1925a). After Einstein, important contributions were made by, most notably, London, Landau, Tisza, Bogoliubov, Penrose, Onsager, Feynman, Lee, Yang, Huang, Beliaev, and Pitaevskii. An important issue has always been the relationship between BEC and superfluidity in liquid helium, an issue that was highly controversial between London and Landau (see Gavroglu, 1995). Works by Bogoliubov, Beliaev, Griffin, and others showed that 
Bose-Einstein condensation gives the microscopic picture behind Landau's "quantum hydrodynamics." BEC is closely related to superconductivity, which can be described as being due to Bose-Einstein condensation of Cooper pairs. Thus Bose-Einstein condensation is at the heart of several macroscopic quantum phenomena.

$\mathrm{BEC}$ is unique in that it is a purely quantum-statistical phase transition, i.e., it occurs even in the absence of interactions. Einstein (1925a) described the transition as condensation "without attractive forces." This makes BEC an important paradigm of statistical mechanics, which has been discussed in a variety of contexts in condensed-matter, nuclear, particle, and astrophysics (Griffin et al., 1995). On the other hand, real-life particles will always interact, and even the weakly interacting Bose gas behaves qualitatively differently from the ideal Bose gas (Huang, 1987). It was believed for quite some time that interactions would always lead to "ordinary" condensation (into a solid) before Bose-Einstein condensation would happen. Liquid helium was the only counterexample, where the light mass and concomitant large zero-point kinetic energy prevents solidification even at zero kelvin. Erwin Schrödinger wrote in 1952 in a textbook on thermodynamics about BEC: "The densities are so high and the temperatures so low-those required to exhibit a noticeable departure [from classical statistics] - that the van der Waals corrections are bound to coalesce with the possible effects of degeneration, and there is little prospect of ever being able to separate the two kinds of effect" (Schrödinger, 1952). What he didn't consider were dilute systems in a metastable gaseous phase!

The quest to realize BEC in a dilute weakly interacting gas was pursued in at least three different directions: liquid helium, excitons, and atomic gases. Experimental (Crooker et al., 1983; Reppy, 1984) and theoretical work (Rasolt et al., 1984) showed that the onset of superfluidity for liquid helium in Vycor has features of dilute-gas Bose-Einstein condensation. At sufficiently low coverage, the helium adsorbed on the porous spongelike glass behaved like a dilute three-dimensional gas. However, the interpretation of these results is not unambiguous (Cho and Williams, 1995).

Excitons, which consist of weakly bound electron-hole pairs, are composite bosons. The physics of excitons in semiconductors is very rich and includes the formation of an electron-hole liquid and biexcitons. As nicely discussed by Wolfe et al. (1995) and Fortin et al. (1995), there are systems where excitons form a weakly interacting gas. However, the initial evidence for Bose-Einstein condensation in $\mathrm{Cu}_{2} \mathrm{O}$ (Lin and Wolfe, 1993) was retracted (O'Hara et al., 1999). Recent work in coupled quantum-well structures is very promising (Butov et al., 2002). When excitons strongly interact with light in a cavity, they form polaritons. In such polariton systems, stimulated scattering and nonequilibrium condensates have been observed recently (Yamamoto, 2000; Saba et al., 2001; Baumberg, 2002).

\section{SPIN-POLARIZED HYDROGEN}

Dilute atomic gases are distinguished from the condensed-matter systems discussed above by the absence of strong interactions. Interactions at the density of a liquid or a solid considerably modify and complicate the nature of the phase transition. Hecht (1959) and Stwalley and Nosanow (1976) used the quantum theory of corresponding states to conclude that spin-polarized hydrogen would remain gaseous down to zero temperature and should be a good candidate to realize BoseEinstein condensation in a dilute atomic gas. These suggestions triggered several experimental efforts, most notably by Silvera and Walraven in Amsterdam, by Greytak and Kleppner at MIT, and by others at Moscow, Turku, British Columbia, Cornell, Harvard, and Kyoto. The stabilization of a spin-polarized hydrogen gas (Cline et al., 1980; Silvera and Walraven, 1980) created great excitement about the prospects of exploring quantumdegenerate gases. Experiments were first done by filling cryogenic cells with the spin-polarized gas, and by compressing it, and since 1985, by magnetic trapping and evaporative cooling. BEC was finally accomplished in 1998 by Kleppner, Greytak and collaborators (Fried et al., 1998). See Greytak and Kleppner (1984), Silvera and Walraven (1986), Greytak (1995), and Walraven (1996) and in particular Kleppner et al. (1999) for a full account of the pursuit of Bose-Einstein condensation in atomic hydrogen. Evidence for a phase transition in two dimensions was reported in 1998 (Safonov et al., 1998).

The work in alkali atoms is based on the work in spinpolarized hydrogen in several respects:

- Studies of spin-polarized hydrogen showed that systems can remain in a metastable gaseous state close to BEC conditions. The challenge was then to find the window in density and temperature where this metastability is sufficient to realize BEC.

- Many aspects of BEC in an inhomogeneous potential (Goldman et al., 1981; Huse and Siggia, 1982; Oliva, 1989), and the theory of cold collision processes (see, for example, Stoof et al., 1988) developed in the 1980s for hydrogen could be applied directly to the alkali systems.

- The technique of evaporative cooling was developed first for hydrogen (Hess, 1986; Masuhara et al., 1988) and then used for alkali atoms.

\section{LASER COOLING}

Laser cooling opened a new route to ultralow temperature physics. Laser cooling experiments, with roomtemperature vacuum chambers and easy optical access, look very different from cryogenic cells with multilayer thermal shielding around them. Also, the number of atomic species that can be studied at ultralow temperatures was greatly extended from helium and hydrogen to all of the alkali atoms, metastable rare gases, several earth-alkali atoms, and others (the list of laser-cooled 
atomic species is still growing). A full account of the relevant laser cooling techniques and their development is given by Arimondo et al. (1992), Metcalf and van der Straten, (1994), and Adams and Riis (1997) and in the 1997 Nobel lectures of Chu (1998), Cohen-Tannoudji (1998), and Phillips (1998).

Some papers and proposals written in the early and mid 1980s, before and during the developments of the basic cooling and trapping techniques, listed quantum degeneracy in a gas as a visionary goal for this new emerging field (Letokhov and Minogin, 1980; Chu et al., 1985; Pritchard, 1986). However, major limitations of laser cooling and trapping were soon identified. Although there is no fundamental low-temperature limit, the final temperature provided by polarization gradient coolingabout ten times the recoil energy-was regarded as a practical limit. Subrecoil laser cooling techniques, especially in three dimensions, were harder to implement, and required long cooling times. The number and density of atoms were limited by inelastic, light-induced collisions (leading to trap loss; see Walker and Feng, 1994; and Weiner, 1995) and by absorption of scattered laser light (Walker et al., 1990), which results in an outward radiation pressure (weakening the trapping potential and limiting the density). Furthermore, since the lowest temperatures could not be achieved at the highest densities (Drewsen et al., 1994; Townsend et al., 1995; 1996), most trapping and cooling techniques reached a maximum phase-space density $n \lambda_{d B}^{3}=10^{-5}$; a value of 2.612 is needed for BEC. This was the situation when the author joined the field of cold atoms in 1990. It was only more recently that major increases in phase-space density were achieved by laser cooling (DePue et al., 1999; Ido et al., 2000; Kerman et al., 2000) but so far laser cooling by itself has not been able to reach BEC.

\section{THE EFFORT AT MIT 1990-1996}

\section{A. Improving laser cooling}

When I teamed up with Dave Pritchard at MIT in 1990 as a postdoc, the initial goal was to build an intense source of cold atoms to study cold collisions and pure long-range molecules. However, Dave and I frequently talked about the limitations in density and temperature of the current techniques and tried to develop ideas on how to get around them. One limitation of magnetic traps is that they can hold atoms only in weak-fieldseeking hyperfine states. Therefore a collision between two trapped atoms can lead to a spin flip, and the Zeeman energy is converted into kinetic energy (dipolar relaxation). This process has been a major limitation to the experiments in atomic hydrogen.

First, we asked ourselves if the inclusion of electric and gravitational fields would allow the stable confinement of atoms in their lowest hyperfine states-but the answer was negative (Ketterle and Pritchard, 1992a). One loophole was time-dependent magnetic fields, and building on an earlier proposal (Lovelace et al., 1985), I designed an experiment to confine sodium atoms with ac magnetic fields which looked feasible. However, we learned that Eric Cornell at Boulder had developed a similar idea and experimentally implemented it (Cornell et al., 1991) - so we left the idea on the drawing board. It wasn't the last time that Eric and I would develop similar ideas independently and almost simultaneously!

Trapping atoms in the lowest hyperfine state was not necessary to accomplish BEC. Already in 1986, Pritchard correctly estimated the rate constants of elastic and inelastic collisions for alkali atoms (Pritchard, 1986). From these estimates one could easily predict that for alkali atoms, in contrast to hydrogen, the so-called good collisions (elastic collisions necessary for the evaporation process) would clearly dominate over the so-called bad collisions (inelastic two- and three-body collisions); therefore evaporative cooling in alkalis would probably not be limited by intrinsic loss and heating processes. However, there was pessimism (Vigué, 1986) and skepticism, and the above-mentioned experimental (Cornell et al., 1991) and theoretical (Ketterle and Pritchard, 1992a) work on traps for strong-field-seeking atoms has to be seen in this context.

In those years, there were some suggestions that timedependent potentials could lead to substantial cooling, but we showed that this was not possible (Ketterle and Pritchard, 1992b). Real cooling needs an open system which allows entropy to be removed from the system-in laser cooling in the form of scattered photons, in evaporative cooling in the form of discarded atoms. Dave and I brainstormed about novel laser cooling schemes. In 1991, at the Varenna summer school, Dave presented a new three-level cooling scheme (Pritchard and Ketterle, 1992). Inspired by these ideas, I developed a scheme using Raman transitions. Replacing the six laser beams in optical molasses by counterpropagating beams driving the Doppler-sensitive Raman transition, we hoped to realize Doppler molasses with a linewidth that was proportional to the optical pumping rate and therefore adjustable. We had started setting up radio frequency (rf) electronics and magnetic shields for Raman cooling when we heard that Mark Kasevich and Steve Chu were working on Raman cooling using laser pulses (Kasevich and Chu, 1992). For this reason, and also because around the same time we had developed the idea for the Dark SPOT (spontaneous force optical trap; see later in this section), we stopped our work on Raman cooling.

Our experimental work in those years focused first on generating a large flux of slow atoms. In my first months at MIT, when I overlapped with Kris Helmerson and Min Xiao, we built a sodium vapor cell magneto-optical trap (MOT). The idea was inspired by the Boulder experiment (Monroe et al., 1990), and our hope was to vastly increase the loading rate by additional frequencies or frequency chirps added to the red side of the $\mathrm{D}_{2}$ resonance line. The idea failed-we first suspected that nearby hyperfine levels of sodium may have adversely interfered, but it was later shown that it didn't work for cesium either (Lindquist et al., 1992) because of the unfavorable duty cycle of the chirp. Still, except for a cryogenic setup which was soon abandoned, it was the first 
magneto-optical trap built at MIT (Dave Pritchard's earlier work on magneto-optical trapping was carried out at Bell Labs in collaboration with Steve Chu's group). We (Michael Joffe, Alex Martin, Dave Pritchard and myself) then put our efforts on beam slowing, and got distracted from pursuing Zeeman slowing by the idea of isotropic light slowing (Ketterle, Martin et al., 1992). In this scheme, atoms are sent through a cavity with diffusely reflecting walls and exposed to an isotropic light field. For red-detuned light the atoms preferentially absorb light from a forward direction and are slowed. The experiment worked very well and it was a lot of fun to do. However, the requirements for laser power and the velocity capture range of this method were inferior to Zeeman slowing, so we decided to build an optimized Zeeman slower.

We adopted the new design by Greg Lafyatis in which the magnetic field increases rather than decreases as in a conventional Zeeman slower (Barrett et al., 1991). We realized that at the magnetic-field maximum it would be possible to apply some additional transverse laser cooling to collimate the slow beam. Michael Joffe, a graduate student, wound a solenoid which had radial access for four extra laser beams. The collimation worked (Joffe et al., 1993), but not as well as we had hoped, and we felt that the small gain was not worth the added complexity. Still, even without collimation, our Zeeman slower provided one of the largest slow-atom fluxes reported until then, and soon after we had a magnetooptical trap with a large cloud of sodium atoms. In hindsight, I am amazed at how many different schemes we considered and tried out, but this may have been necessary to distill the best approach.

The 1991 Varenna summer school on laser cooling was memorable to me for several reasons. I had joined the field of cold atoms just a year earlier, and there I met many colleagues for the first time and established longlasting relationships. I still have vivid memories of one long afternoon when Dave Pritchard and I sat outside the meeting place, which offered a spectacular view of Lake Como, and brainstormed about the big goals of our field and how to approach them. Dave's encouragement was crucial to me and helped to increase my selfconfidence in my new field of research. We considered options and strategies on how to combine laser cooling and evaporative cooling, something which had been on our mind for some time.

Following the example of the spin-polarized hydrogen experiment at MIT (Masuhara et al., 1988), evaporation could be done in a magnetic trap using rf-induced spin flips, as suggested by Pritchard and collaborators (Pritchard, Helmerson, and Martin, 1989). Magnetic traps and laser cooling had already been used simultaneously in the first experiments on magnetic trapping at NIST (Migdall et al., 1985) and MIT (Bagnato et al., 1987), and on Doppler cooling of magnetically trapped atoms at MIT (Prichard et al., 1989; Helmerson et al., 1992). In 1990, a magnetic trap was loaded from a magnetooptical trap and optical molasses in Boulder (Monroe et al., 1990). The laser cooling route to BEC was sum- marized by Monroe, Cornell, and Wieman (1992). So most of the pieces to get to BEC were known in 1990, but there was doubt about whether they would fit together.

Laser cooling works best at low densities where light absorption and light-induced collisions are avoided, whereas evaporative cooling requires a high collision rate and high density. The problem is the much higher cross section for light scattering of $\sim 10^{-9} \mathrm{~cm}^{2}$, while the cross section for elastic scattering of atoms is a thousand times smaller. In hindsight, it would have been sufficient to provide tight magnetic compression after laser cooling and an extremely good vacuum to obtain a lifetime of the sample that is much longer than the time between collisions, as demonstrated at Rice University (Bradley et al., 1995). However, our assessment was that one major improvement had to be done to laser cooling to bridge the gap in density between the two cooling schemes. Dave and I discussed possibilities on how to circumvent the density-limiting processes in magnetooptical traps. We considered coherent population trapping schemes in which atoms are put into a coherent superposition state which does not absorb the light. We developed some ideas on how atoms near the center of the trap would be pumped into such a dark state, but the numbers were not too promising. A few months later, a simple idea emerged. If the so-called repumping beam of the magneto-optical trap would have a shadow in the center, atoms would stay there in the lower hyperfine state and not absorb the trapping light, which is near resonant for atoms in the upper hyperfine state. In a MOT, the density is limited by losses due to excitedstate collisions and by multiple scattering of light, which results in an effective repulsive force between atoms. When atoms are kept in the dark, the trapping force decreases by a factor which is proportional to the probability of the atoms to be in the resonant hyperfine state. However, the repulsive force requires both atoms to be resonant with the light and decreases with the square of this factor. Therefore there is net gain in confinement by keeping atoms in the dark. Of course, there is a limit to how far you can push this concept, which is reached when the size of the cloud is no longer determined by the balance of trapping and repulsive forces, but by the finite temperature of the cloud.

The gain in density of this scheme, called Dark SPOT, over the standard MOT is bigger when the number of trapped atoms is large. So in 1992, we tweaked up the MOT to a huge size before we implemented the idea. It worked almost immediately, and we got very excited about the dark shadows cast by the trapped atoms when they were illuminated by a probe beam. We inferred that the probe light had been attenuated by a factor of more than $e^{-100}$ (Ketterle et al., 1993a). This implied that we had created a cloud of cold atoms with an unprecedented combination of number and density.

\section{B. Combining laser cooling and evaporative cooling}

The following weeks and months were quite dramatic. What should we do next? Dave Pritchard had planned 
to use this trap as an excellent starting point for the study of cold collisions and photoassociation-and indeed other groups had major successes along these lines (Heinzen, 1999; Weiner et al., 1999). But there was also the exciting prospect of combining laser cooling with evaporative cooling. We estimated the elastic collision rate in the Dark SPOT trap to be around $100 \mathrm{~Hz}$ (Ketterle et al., 1993a) which appeared to be more than sufficient to start runaway evaporation in a magnetic trap. After some discussions, the whole group decided to go for the more ambitious and speculative goal of evaporative cooling. It was one of those rare moments where suddenly the whole group's effort gets refocused. Even before we wrote the paper on the Dark SPOT trap, we placed orders for essential components to upgrade our experiment to ultrahigh vacuum and to magnetic trapping. All resources of the lab were now directed towards the evaporative cooling of sodium. The Dark SPOT trap was a huge improvement towards combining high atom number and high density in laser cooling. It turned out to be crucial to the BEC work both at Boulder (Anderson et al., 1995) and at MIT (Davis, Mewes, Andrews, et al., 1995) and seems to be still necessary in all current BEC experiments with sodium, but not for rubidium.

The next step was the design of a tightly confining magnetic trap. We decided to use the spherical quadrupole trap, which simply consists of two opposing coilsthis design was used in the first demonstration of magnetic trapping (Migdall et al., 1985). We knew that this trap would ultimately be limited by Majorana flops in the center of the trap where the magnetic field is zero. Near zero magnetic field, the atomic spin doesn't precess fast enough to follow the changing direction of the magnetic field - the result is a transition to another Zeeman sublevel which is untrapped, leading to trap loss. We estimated the Majorana flop rate, but there was some uncertainty about the numerical prefactor. Still, it seemed that Majorana flops would only become critical after the cloud had shrunk due to evaporative cooling, so they shouldn't get in the way of demonstrating the combination of laser cooling and evaporative cooling. After Michael Joffe presented our approach with the quadrupole trap at the QELS meeting in 1993, Eric Cornell informed me that he had independently arrived at the same conclusion. In 1993, my group reported at the OSA meeting in Toronto the transfer of atoms from the Dark SPOT trap into a magnetic trap, and the effects of truncation of the cloud using rf induced spinflips (Ketterle et al., 1993b).

At about this time, I joined the MIT faculty as assistant professor. Dave Pritchard made the unprecedented offer that if I stayed at MIT he would hand over to me the existing lab, including two grants. To make sure that I would receive the full credit for the work towards BEC, he decided not to stay involved in a field he had pioneered and gave me full responsibility and independence. Dave told me that he wanted to focus on his other two experiments, the single-ion mass measurement and the atom interferometry, although what he gave up was his "hottest" research activity. Even now, I am moved by his generosity and unusual mentorship. The two graduate students on the project, Ken Davis and Marc-Oliver Mewes, who had started their Ph.D.'s in 1991 and 1992, respectively, deliberated whether they should stay with Dave Pritchard and work on one of his other experiments, or continue their work on BEC in a newly formed group headed by a largely unknown assistant professor. They both opted for the latter and we could pursue our efforts without delay, along with Michael Andrews, who joined the group in the summer of 1993.

For a few months we got distracted from our goal of evaporative cooling. Our optical molasses temperatures were higher than those reported by the NIST group (Lett et al., 1989), and we felt that we had to learn the state of the art before we could advance to even lower temperatures. We suspected that the higher density of atoms played a role, but we had to improve our technique of temperature measurements. Our goal was to characterize the interplay of parameters in "dark" molasses where most of the atoms are pumped into the dark hyperfine state. It was also a good project for the graduate students to hone their skills and develop independence. After a few months we had made some progress, but I became concerned about the delay and the competition from Boulder. We decided to drop the project and resume our work on evaporative cooling. Up to the present day, we have never implemented accurate diagnostics for the temperature obtained in laser cooling-it was just not important.

In the spring of 1994, we saw first evidence for an increase in phase-space density by evaporative cooling. We reported these results at an invited talk at the International Quantum Electronics Conference (IQEC) in May 1994. At the same meeting, the Boulder group reported similar results and the limitations due to the Majorana flops as the temperature was reduced. It was clear that the next step was an improvement of the magnetic trap, to trap atoms at a finite bias field which would suppress the Majorana flops. During the meeting, I came up with the idea of plugging the hole with a focused laser beam: a blue-detuned laser beam focused onto the zero-magnetic-field point would exert repulsive dipole forces onto the atoms and keep them away from this region (Fig. 3). This idea seemed so obvious to me that I expected the Boulder group to come up with something similar. It was only at the next conference (ICAP 1994) in Boulder (Davis et al., 1994), when I presented our approach, that I learned about Eric Cornell's idea of suppressing Majorana flops with a rapidly rotating magnetic field-the so-called TOP trap (Petrich et al., 1995). However, we didn't implement the optical plug immediately. We wanted first to document our observation of evaporative cooling. We realized that our fluorescence diagnostics were inadequate and implemented absorption imaging which is now the standard technique for observing Bose-Einstein condensation. In those days, we focused on direct imaging of the trapped cloud (without ballistic expansion), and Michael Andrews and MarcOliver Mewes developed a sophisticated computer code to simulate absorption images in inhomogeneous mag- 


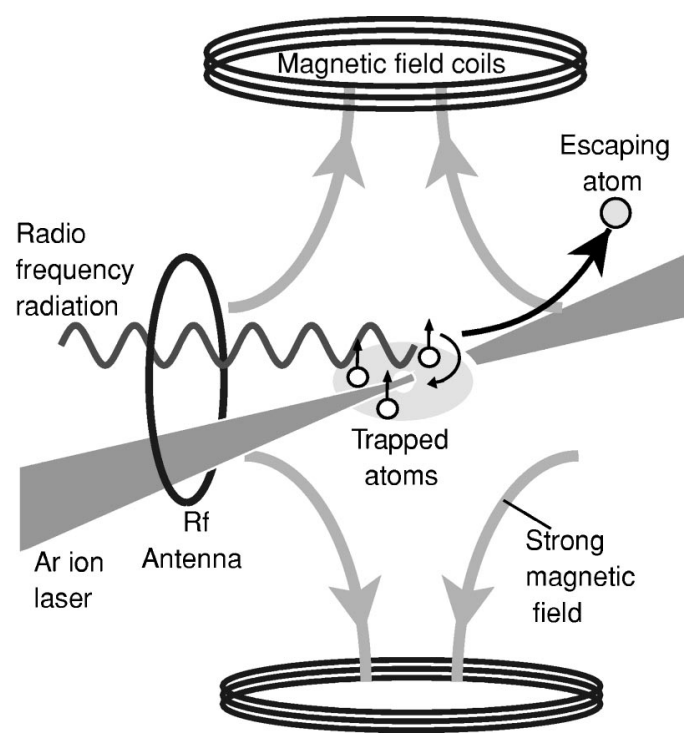

FIG. 3. Experimental setup for cooling atoms to Bose-Einstein condensation. Sodium atoms are trapped by a strong magnetic field, generated by two coils. In the center, the magnetic field vanishes, which allows the atoms to spin flip and escape. Therefore the atoms are kept away from the center of the trap by a strong (3.5-W) argon ion laser beam ("optical plug"), which exerts a repulsive force on the atoms. Evaporative cooling is controlled by radio-frequency radiation from an antenna. The rf selectively flips the spins of the most energetic atoms. The remaining atoms rethermalize (at a lower temperature) by collisions among themselves. Evaporative cooling is forced by lowering the rf frequency.

netic fields. We thought that this would be a useful tool, but we rapidly advanced to much lower temperatures where the inhomogeneous Zeeman shifts were smaller than the linewidth, and never needed the code again after our first paper on evaporative cooling (Davis, Mewes, Joffe, et al., 1995).

In late 1994, we had a "core meltdown." The magnetic trap was switched on without cooling water, and the silver solder joints of the coils melted. Since in those days the magnetic coils were mounted inside the vacuum chamber, we had a catastrophic loss of vacuum and major parts of our setup had to be disassembled. I will never forget the sight of coils dripping with water behind a UHV viewport. This happened just a few hours before MIT's president, Charles Vest, visited our lab to get firsthand information on some of the research done on campus. He still remembers this event. We had lost weeks or months of work in a very competitive situation. I was despondent and suggested to the group that we go out for a beer and then figure out what to do, but the students immediately pulled out the wrenches and started the repair. I was moved to see their dedication and strength, even at this difficult time. We replaced the magnetic trap by a much sturdier one. This turned out to be crucial for the implementation of the plugged trap where the precise alignment of a laser beam relative to the magnetic field center was important. So in hindsight the disaster may not have caused a major delay.

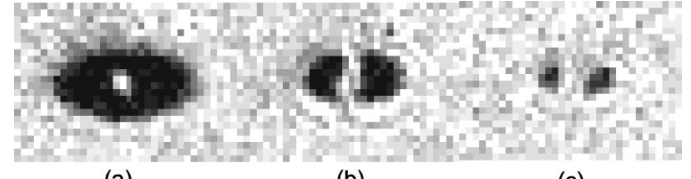

(a)

(b)

(c)

FIG. 4. Absorption images of atom clouds trapped in the optically plugged trap. Cloud (a) is already colder than was attainable without the "plug" (Ar ion laser beam). Cloud (b) shows the breakup of the cloud into two "pockets" in the two minima of the potential. The size of cloud (c) reached the optical resolution of the imaging system $(\leqslant 10 \mu \mathrm{m})$ still absorbing $90 \%$ of the probe light. This sets an upper bound on temperature $(\leqslant 10 \mu \mathrm{K})$ and a lower bound on density (5 $\left.\times 10^{12} \mathrm{~cm}^{-3}\right)$.

In early 1995, I had to tell my three graduate students that we were rapidly using up startup money and urgently needed one of our two pending proposals approved. Otherwise we would not be able to continue spending money in the way we had done until then and would slow down. Fortunately, in April 1995, the NSF informed me that my proposal was funded. It is interesting to look at some of the reviewers comments now, seven years later: "It seems that vast improvements are required [in order to reach BEC]...the current techniques are so far from striking range for BEC that it is not yet possible to make... an assessment..."; "The scientific payoffs, other than the importance of producing a BEC itself, are unclear." And a third reviewer: "...there have been few specific (or realistic) proposals of interesting experiments that could be done with a condensate." Despite the skepticism, all reviewers concluded that the proposed "experiments are valuable and worth pursuing." After we received the funding decision, the whole group celebrated with dinner, and a fourth graduate student (Dallin Durfee), who had expressed his interest already months earlier, could finally be supported.

In late December 1994, our paper on evaporative cooling was submitted, and we were free to focus on plugging the hole. We had to learn how to align a powerful argon ion laser beam and image it through many attenuators without major distortions. When the plug was aligned, the result was spectacular (Fig. 4). We could immediately cool down to lower temperatures and keep many more atoms. During evaporation, the cloud became so cold and small that we couldn't resolve it any more. The highest phase-space density measured was a factor of 30 below BEC, but we may have been even closer. We had only a few runs of the experiment before we ran into severe vacuum problems. We focused initially on spatial imaging and became limited by resolution, whereas ballistic expansion and time-of-flight imaging would not have suffered from this limitation. We also thought that BEC would be accomplished at lower densities and in larger clouds, so we worked on adiabatic decompression and ran into problems with the zero of the magnetic field moving away from the plug.

In those months, we were plagued by vacuum problems. The coils inside the vacuum showed some strange outgassing behavior and the vacuum slowly deterio- 
rated. We went through several bakeouts of the ultrahigh-vacuum chamber in the spring and summer of 1995. Furthermore, Ken Davis had to write his Ph.D. thesis and stopped working in the lab. It is interesting to recall my assessment of the field in those months; I didn't realize that BEC was just around the corner. In Tom Greytak's and Dan Kleppner's group the BEC transition was approached to within a factor of 3.5 in temperature in 1991 (Doyle et al., 1991), but it took several more years to advance further. So I prepared for a long haul to cover the last order of magnitude to BEC.

By this time, the group was reinforced by Dan Kurn (now Dan Stamper-Kurn), a graduate student, and Klaasjan van Druten, my first postdoc. After months of working on vacuum and other problems, we were just ready to run the machine again when we heard about the breakthrough in Boulder in June of 1995 (Anderson et al., 1995). We feverishly made several attempts with traps plugged by focused laser beams and light sheets, and tried different strategies of evaporation without success. The clouds disappeared when they were very cold. We conjectured that some jitter of the laser beam was responsible, and when accelerometers indicated vibrations of our vacuum chambers, we immediately decided to eliminate all turbo and mechanical pumps. Unfortunately, when we were exchanging the turbo pump on our oven chamber against an ion pump, we caused a leak in the ultrahigh-vacuum part and had to go through another long bakeout. We also implemented a pointing stabilization for the optical plug beam. But when we finally obtained BEC, we realized that it didn't improve the cooling.

These were difficult months for me. The Rice group had cooled lithium to quantum degeneracy (Bradley et al., 1995). A new subfield of atomic physics was opening up, and I was afraid that our approach with sodium and the plugged trap would not be successful and we would miss the excitement. I considered various strategies. Several people suggested that I adopt the successful TOP trap used at Boulder. But I had already started to study several possible configurations for magnetic confinement. I realized that a highly elongated IoffePritchard trap with adjustable bias field could provide a good confinement that was equivalent or superior to the TOP trap. Around August 1995, Dan Kurn worked out an optimized configuration, which was the cloverleaf winding pattern (Mewes et al., 1996a). I considered having the whole group work on this new approach, but several in my group wanted to give the plugged trap a few more attempts and at least characterize how far we could approach BEC with our original approach. Fortunately, we followed that suggestion-it is always a good idea to listen to your collaborators.

\section{BEC in sodium}

This was the situation on September 29, 1995, when we observed BEC in sodium for the first time. The goal of the run was to measure the lifetime of the trapped atoms and characterize possible heating processes. For

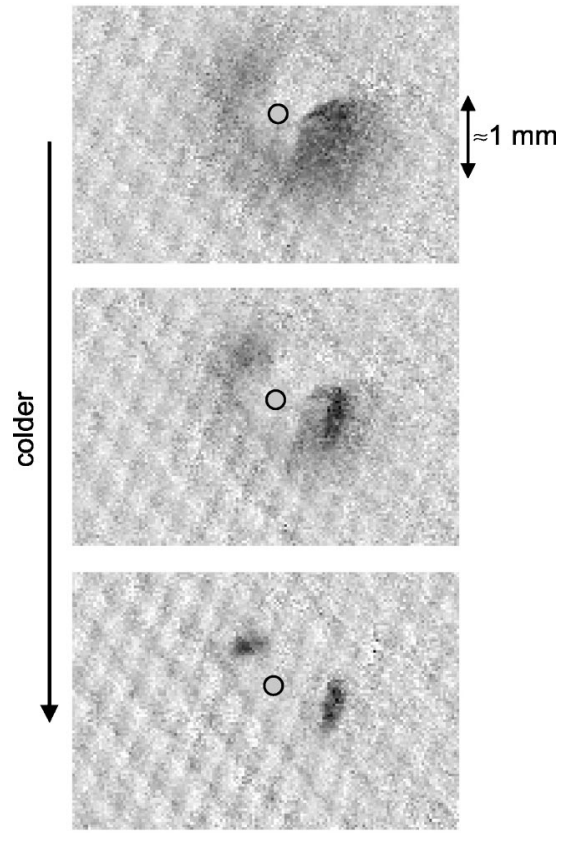

FIG. 5. Time-of-flight absorption images of some of the first condensates produced at MIT in the night of September 29, 1995. After the magnetic quadrupole trap was switched off, the atom cloud expanded ballistically. However, since the optical plug (indicated by black circles) could not be turned off at the same time, it distorted the expanding cloud. Still, as the temperature was lowered from top to bottom, a distinctly sharp shadow appeared marking the presence of a condensate.

our ultrahigh-vacuum pressure, rather slow evaporation should have been most efficient, but we found out that faster evaporation worked much better. This was a clear sign for some other loss or heating process, e.g., due to fluctuations in the position of the plug. Around 11:30 p.m., an entry in the lab book states that the lifetime measurements were not reliable, but they indicated lifetimes around ten seconds, enough to continue evaporation. Fifteen minutes later we saw some dark spots in time-of-flight absorption images, but they were quite distorted since the optical plug beam, which we couldn't switch off, pushed atoms apart during the ballistic expansion (Fig. 5). Still, the sudden appearance of dark spots meant groups of atoms with very small relative velocity. For the next few hours, we characterized the appearance of those spots, but then decided that further progress required an acousto-optical modulator to switch off the optical plug. Between 4:00 and 5:30 in the early morning, we installed optics and rf electronics and were finally able to switch off the argon ion laser beam during ballistic expansion. A few minutes later, we observed the bimodal distributions that are now the hallmark of BEC. The lab book of this night captured the excitement of the moment (Fig. 6).

Those first measurements were done by imaging the atoms in the lower hyperfine $(F=1)$ state. For the next run, which took place a few days later, we prepared optical pumping and imaging on the cycling $F=2$ transition, and obtained a much better signal-to-noise ratio in our images. The occurrence of BEC was very dramatic 


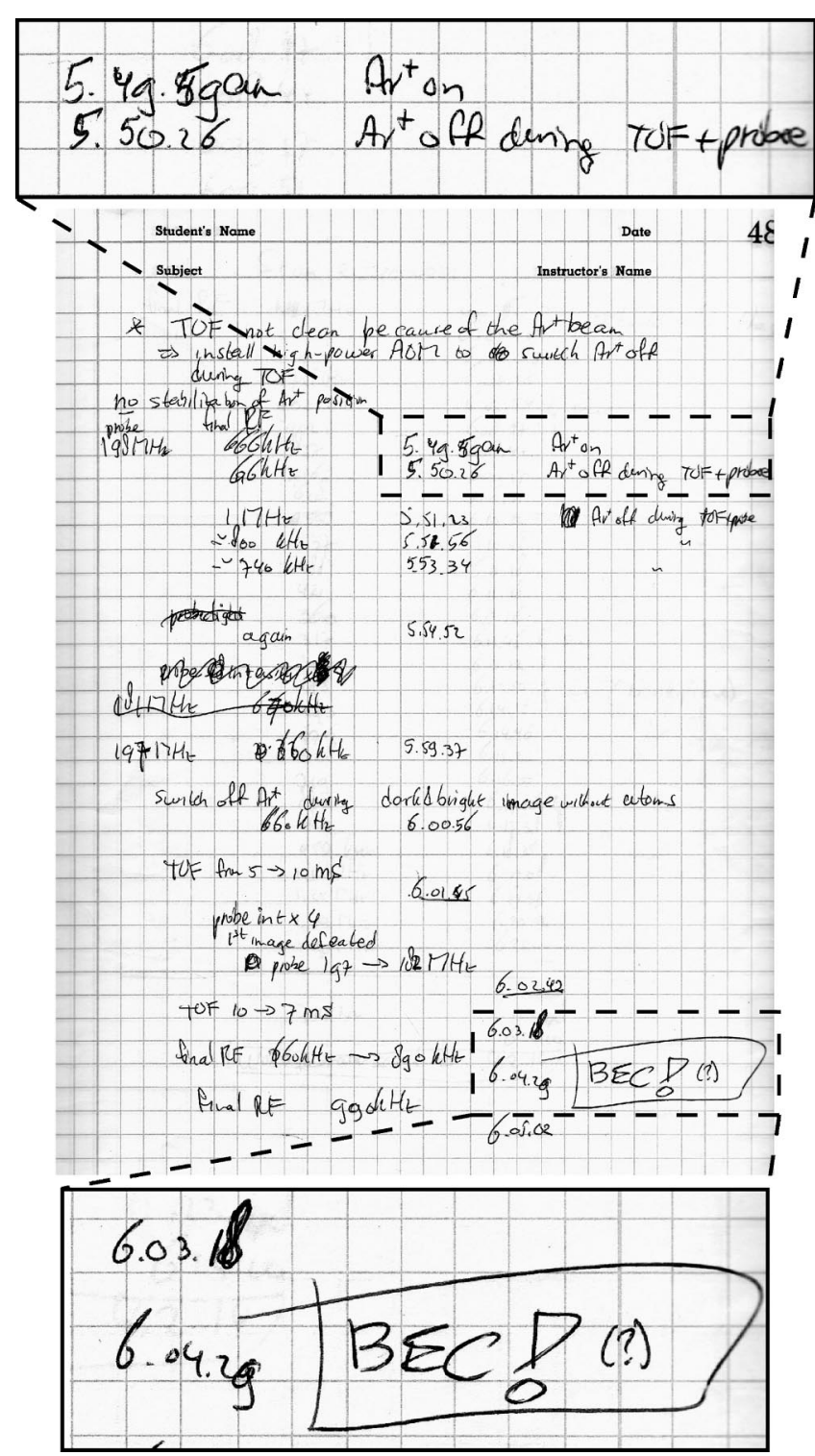

FIG. 6. One page of the lab book during the night of September 29, 1995, when BEC was first observed at MIT. The handwriting is by Klaasjan van Druten. At 5:50 a.m., we had installed a new acousto-optical modulator to switch off the optical plug (Ar ion laser beam). Fifteen minutes later, we had the first definitive evidence for BEC in sodium.

(Fig. 7). Our animated rendering of the data obtained in that run (done by Dallin Durfee) became well known (see Durfee and Ketterle, 1998). We had obtained condensates with 500000 atoms, 200 times more than in Boulder, with a cooling cycle (of only nine seconds) 40 times shorter. Our paper was quickly written and submitted only two weeks after the experiment (Davis, Meewes, Andrews, et al., 1995).

In my wildest dreams I had not assumed that the step from evaporative cooling to BEC would be so fast. Figure 8 shows how dramatic the progress was after laser and evaporative cooling were combined. Within less than two years, the number of alkali atoms in a single quantum state was increased by about 12 orders of magnitude - a true singularity demonstrating that a phase transition was achieved!
MIT with its long tradition in atomic physics was a special place to pursue the BEC work. The essential step was the combination of laser cooling and evaporative cooling. My next-door neighbors in Building 26 at MIT have been Dave Pritchard, a pioneer in laser cooling who conceived the magneto-optical trap, and Dan Kleppner, who together with Harald Hess and Tom Greytak conceived and realized evaporative cooling (see Fig. 9). I feel privileged for the opportunity to combine their work and take it to the next level. It is hard to overestimate the roles which Dave Pritchard and Dan Kleppner have played for modern atomic physics. The family tree of atomic physicists (Fig. 10) shows some of the remarkable physicists who were trained and inspired by them.

Looking back, it seems that many techniques such as the Dark SPOT, compressed MOT (Petrich et al., 1994), the TOP trap and the optically plugged trap were critical for first demonstrating BEC, but by no means indispensable. This is best illustrated by the experiment at Rice, which used only Doppler cooling to load the magnetic trap-a technique which had been developed in the 1980s. The collision rate was slow, but an excellent vacuum made a very slow evaporation process possible (Bradley et al., 1995). So in hindsight, BEC in alkali gases did not require major innovations in cooling and trapping. It merely required enough optimism to risk a few years in the attempt to combine laser and evaporative cooling. Such an attempt needed a few years of very focused work as it involved the integration of several technologies that were not standard in the field, including ultrahigh vacuum, sensitive CCD cameras and image processing, high-current power supplies for magnetic traps, and flexible computer control of a multistep cooling and detection process. Figure 11 compares a stateof-the-art laser cooling experiment in 1993 to a BEC experiment in 2001 using the same vacuum apparatus in the same laboratory at MIT. A lot of components have been added, and I continue to be impressed by my collaborators, who now handle experiments far more complex than I did some five years ago.

\section{The cloverleaf trap}

After our first observation of BEC, we made the right decision for the wrong reason. We expected many other groups to quickly upgrade their laser cooling experiments to magnetic trapping and evaporative cooling, and to join in during the next few months. Nobody expected that it would take almost two years before the next groups succeeded in reaching BEC (the groups of Dan Heinzen, Lene Hau, Mark Kasevich, and Gerhard Rempe followed in 1997). I was concerned that our plugged trap would put us at a disadvantage since the trapping potential strongly depended on the shape and alignment of the laser focus. So we decided to install the cloverleaf trap instead and discontinue our plugged trap after only two experimental BEC "runs."

Since we didn't want to break the vacuum, we installed the new trap in an unfavorable geometry. The 


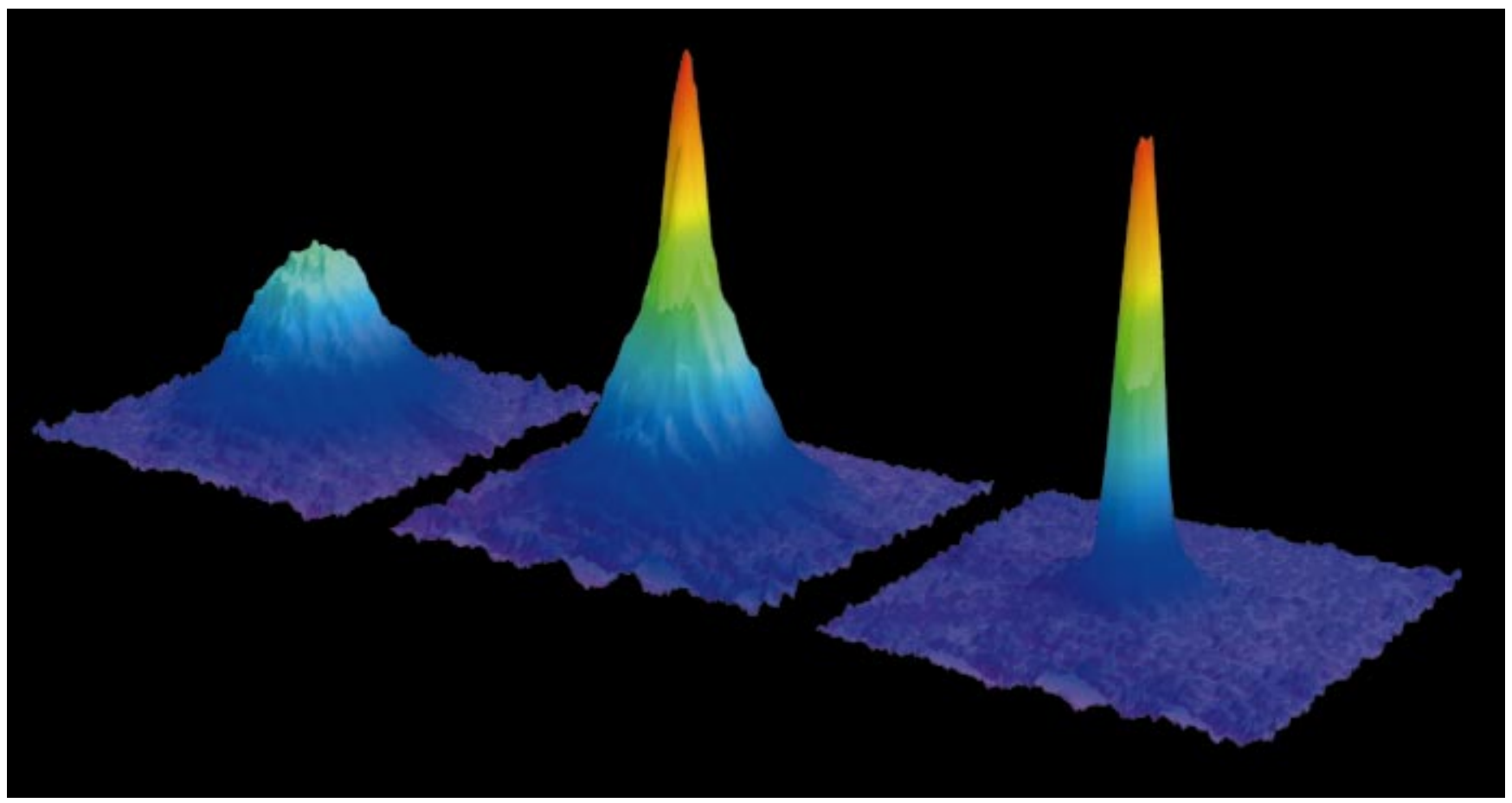

FIG. 7. Observation of Bose-Einstein condensation by absorption imaging. Shown is absorption vs two spatial dimensions. The Bose-Einstein condensate is characterized by its slow expansion observed after $6 \mathrm{~ms}$ time of flight. The left picture shows an expanding cloud cooled to just above the transition point; middle: just after the condensate appeared; right: after further evaporative cooling has left an almost pure condensate. The total number of atoms at the phase transition is about $7 \times 10^{5}$, the temperature at the transition point is $2 \mu \mathrm{K}$ [Color].

magnet coils for the plugged trap were oriented vertically in reentrant flanges, and when we replaced them with cloverleaf coils, the weakly confining axis of the Ioffe-Pritchard trap was vertical. In such a geometry, the gravitational sag would reduce the efficiency of rfinduced evaporation since atoms would only evaporate

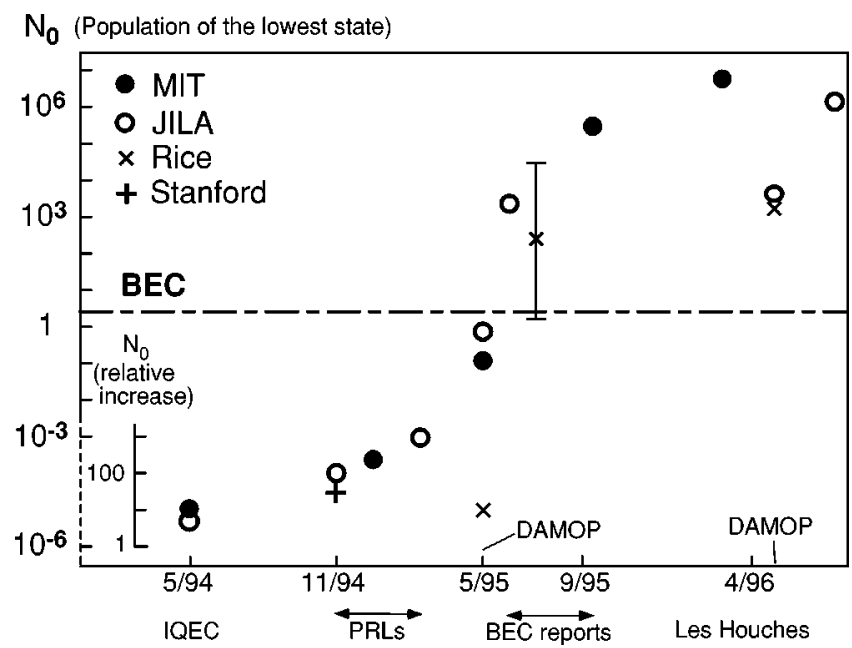

FIG. 8. Progress in evaporative cooling of alkali atoms up to 1996. The number of atoms in the lowest quantum state is proportional to the phase-space density and has to exceed a critical number of 2.612 to achieve Bose-Einstein condensation. For $N_{0}<10^{-3}$, the increase in phase-space density due to evaporation is plotted. For the Rice result of July 1995 see Bradley et al. (1995) and the erratum (Bradley et al., 1997). at the bottom of the cloud (Ketterle and van Druten, 1996; Surkov et al., 1996). But before breaking the vacuum and reorienting the coils, we wanted to see the limitation. Around December 1995, when we were just starting to look at the efficiency of evaporation, we lost the vacuum once again due to a cracked ceramic part in an electric feedthrough and decided to reorient the whole experiment, with the weakly confining axis of the trap now aligned horizontally. Since that time, now more than six years, the machine has been under vacuum. This is in sharp contrast to the conditions in 1995, when we had to open the chamber, pump down, and bake out every couple of months. Finally, we had learned from our previous mistakes and developed a very systematic procedure for pumpdowns and bakeouts.

I still remember the night of March 13, 1996, when the experiment was up and running, and Klaasjan van Druten and I had fine-tuned the bias field of the magnetic trap, so that the switchover to the new magnetic trap was finally completed. It was already after midnight, too late to start some serious work, when Klaasjan asked half jokingly why don't we just try to get BEC. Without knowing what our temperatures and densities were, without having ever measured the trap frequencies, we played around with the rf sweep that determines the cooling trajectory, and a condensate showed up around 2:10 a.m. We were relieved since we hadn't produced condensates for almost half a year, but also the ease at which we got the condensate in a new trap told us our setup was robust and that we were ready to 


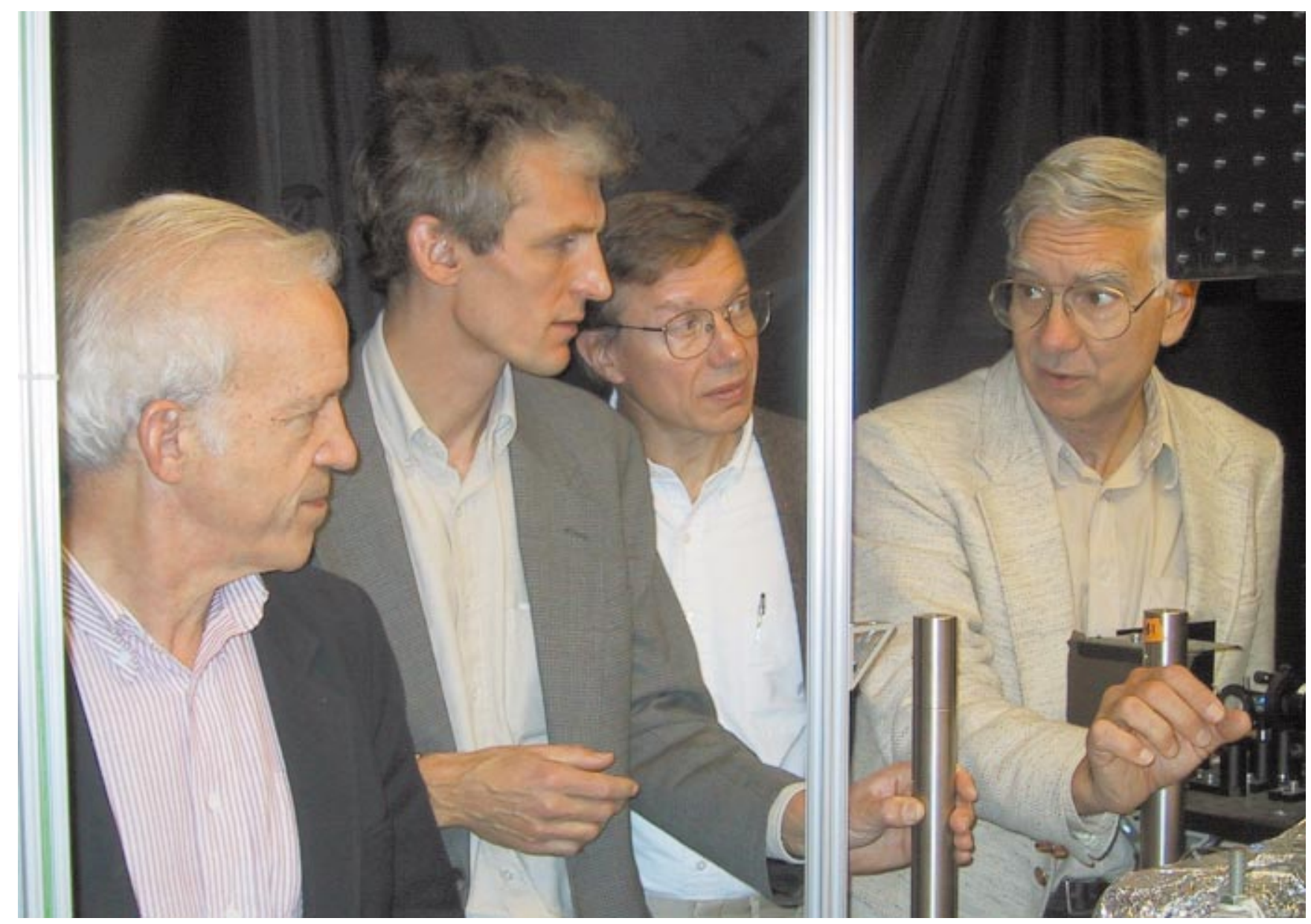

FIG. 9. MIT faculty in ultralow-temperature atomic physics. Dan Kleppner, W.K., Tom Greytak, and Dave Pritchard look at the latest sodium BEC apparatus [Color].

switch from engineering cooling schemes and traps to the study of the condensate. The cloverleaf trap and other winding patterns for the Ioffe-Pritchard configuration are now used by almost all BEC experiments. Figure 12 shows the experimental setup during those days.

Why hadn't we considered this trap earlier and avoided the detours with the quadrupole trap, Majorana

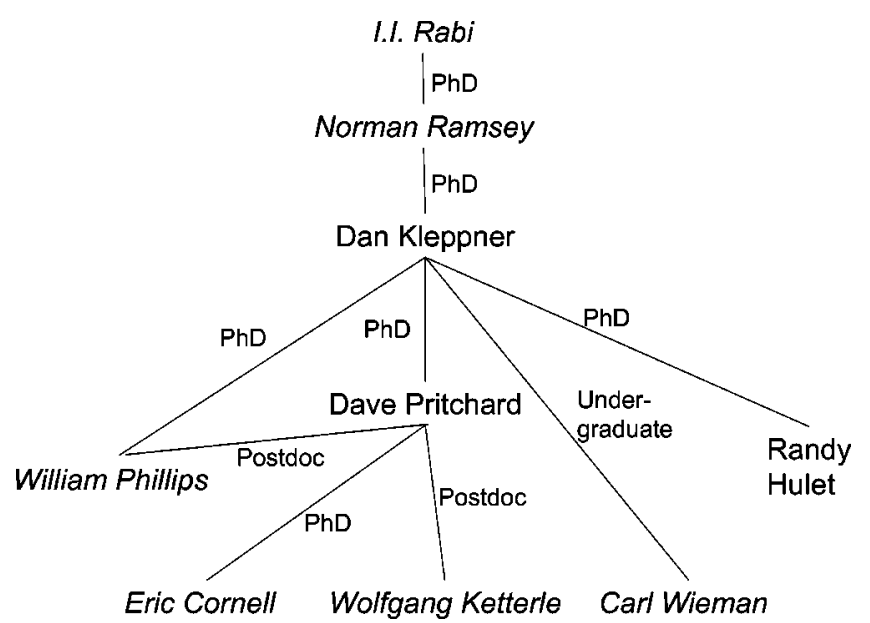

FIG. 10. Family tree of atomic physicists. People with names in italics are Nobel laureates. flops, and plugging the hole? First, the quadrupole trap was simpler to build, and it allowed us to pursue evaporative cooling faster. Second, we initially favored the quadrupole trap based on an analysis which shows that confinement by a linear potential is much stronger than by the quadratic potential of the Ioffe-Pritchard configuration (Ketterle, Durfee, and Stamper-Kurn, 1999). However, a very elongated Ioffe-Pritchard trap provides effectively linear confinement in the two radial directions, and it was only in 1995 that I realized that it would be easy to adiabatically deform the round laser-cooled cloud to such an elongated shape.

The next weeks were exciting and dramatic: we implemented dispersive imaging and saw for the first time the condensate in the trap. We could take images nondestructively and recorded two sequential images of the same condensate. After year-long concerns of how fragile and sensitive the condensate would be once created, it was an overwhelming experience to observe the condensate without destroying it. Figure 13 shows a spatial image of a condensate; it was taken in nondestructive dispersive imaging. We first implemented dispersive imaging using the dark-ground technique (Andrews et al., 1996), but soon upgraded to phase-contrast imaging, which was the technique used to record the figure. 


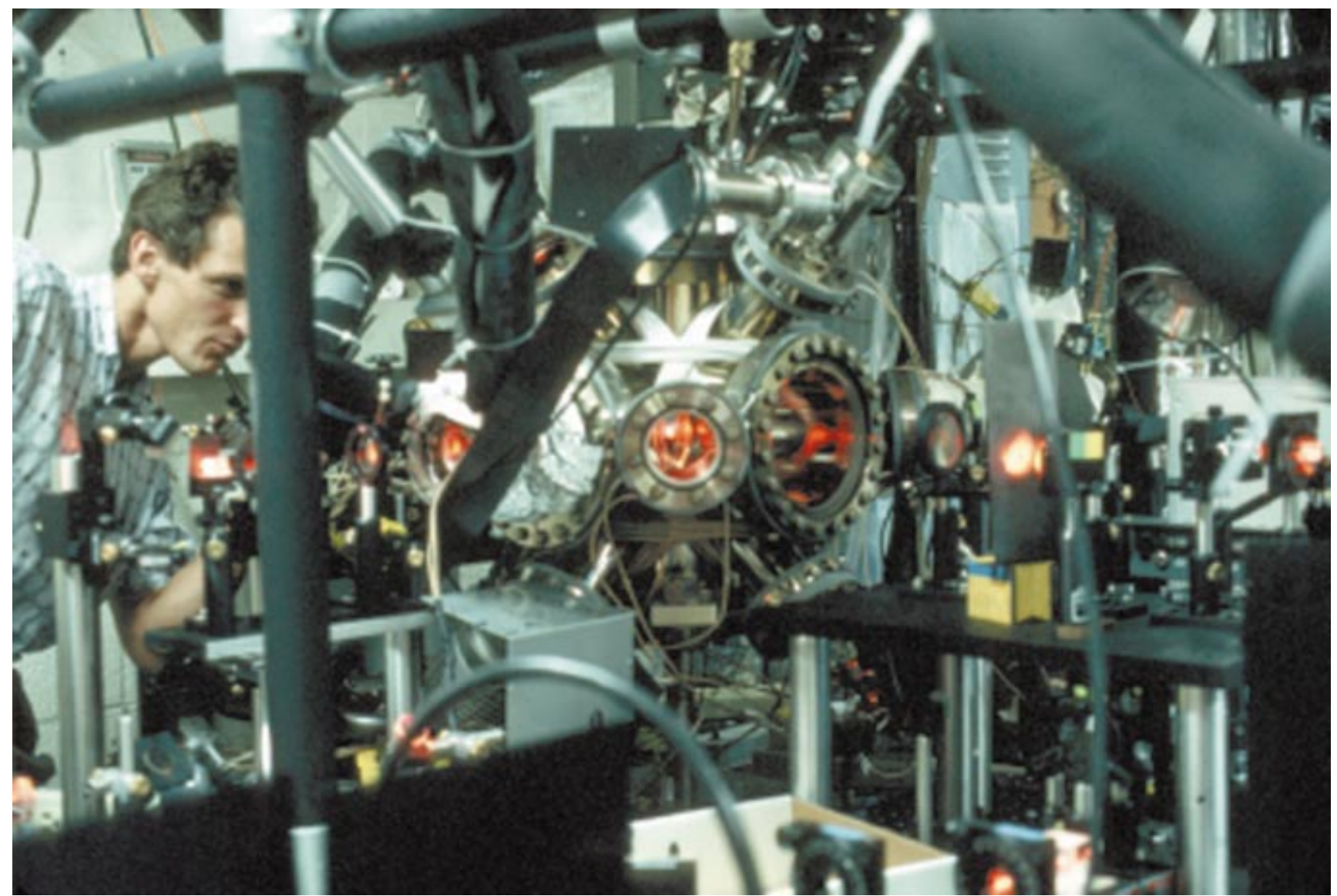

FIG. 11. Comparison of a laser cooling and BEC experiment. The first photograph shows the author in 1993 working on the Dark SPOT trap. In the following years, this laser cooling experiment was upgraded to a BEC experiment. The second photograph shows the same apparatus in 2001 after many additional components have been added [Color].

In the first week of April 1996, there was a workshop on "Collective effects in ultracold atomic gases" in Les Houches, France, where most of the leading groups were represented. It was the first such meeting after the summer of 1995, and it was not without strong emotions that I reported our results. Since no other experimental group had made major progress in BEC over the last few months, it was our work which provided optimism for further rapid developments.

\section{E. Interference between two condensates}

After we got BEC in the cloverleaf trap, both the machine and the group were in overdrive. After years of building and improving, frequent failures and frustration, it was like a phase transition to a situation where almost everything worked. Within three months after getting a condensate in the cloverleaf trap we had written three papers on the new trap and the phase transition (Mewes et al., 1996a), on nondestructive imaging (Andrews et al., 1996), and on collective excitations (Mewes et al., 1996b). Klaasjan van Druten left the group, shortly after Christopher Townsend had joined us as a postdoc. As the next major goal, we decided to study the coherence of the condensate. With our optical plug, we had already developed the tool to split a con- densate into two halves and hoped to observe their interference, which would be a clear signature of the longrange spatial coherence.

Around the same time, the idea came up to extract atoms from the condensate using rf induced spin flipsthe rf output coupler. Some theorists regarded an output coupler as an open question in the context of the atom laser. I suggested to my group that we could simply pulse on the radio-frequency source that was already used during evaporation, and couple atoms out of the condensate by flipping their spin to a nontrapped state (Fig. 14). The experiment worked the first time we tried it (but the quantitative work took awhile; Mewes et al., 1997). I have never regarded the output coupler as one of our major accomplishments because it was so simple, but it had impact on the community and nobody has ever since regarded outcoupling as a problem!

In July 1996, we had the first results on the rf output coupler, and also saw the first fringes when two condensates were separated with a sheet of green light and overlapped in ballistic expansion. I was in Australia for vacation and for the IQEC conference in Sydney. By e-mail and telephone I discussed with my group the new results. The fringes were most pronounced when the condensates were accelerated into each other by removing the light sheet shortly before switching off the mag- 


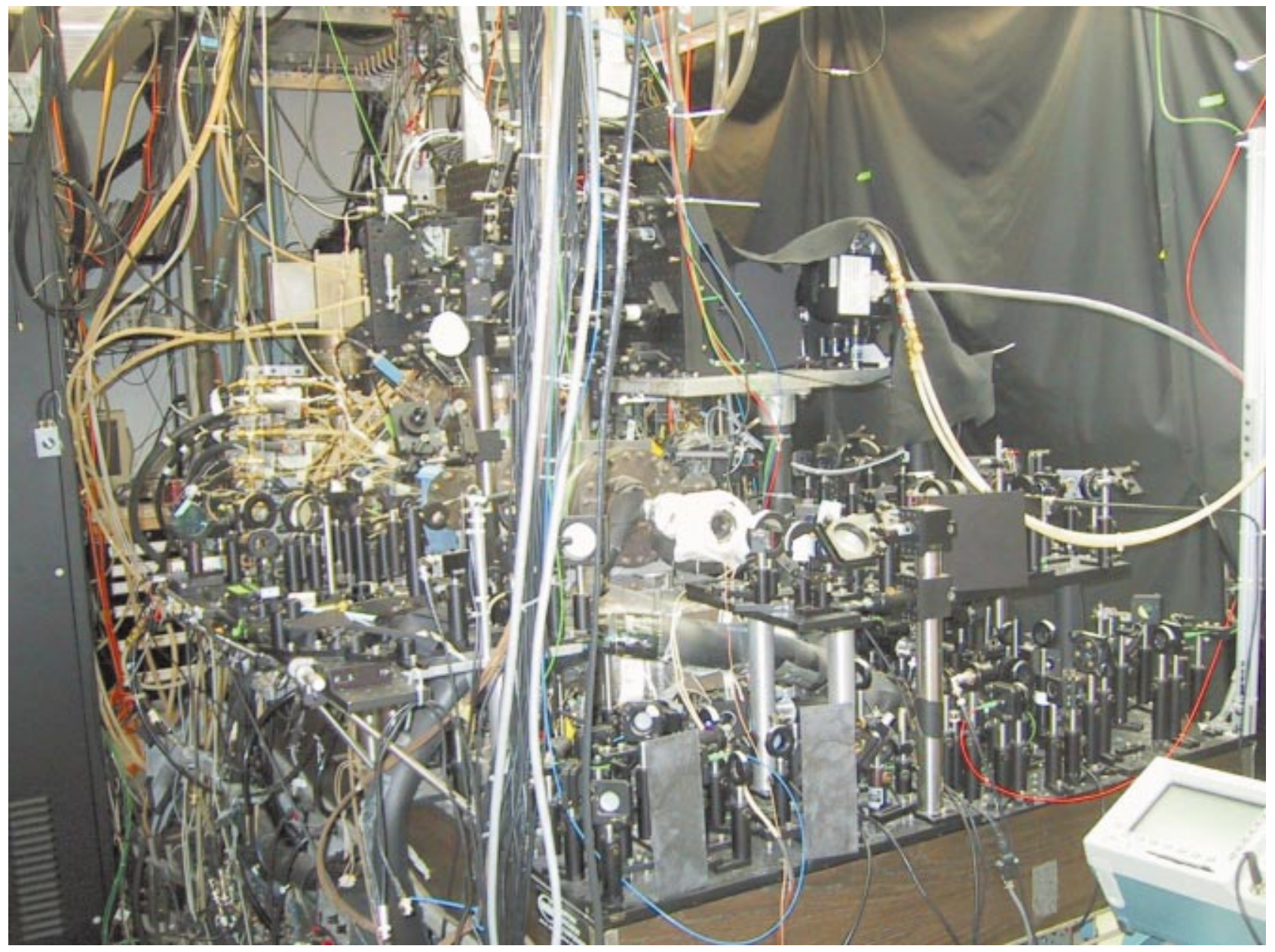

FIG. 11. (Continued.)

netic trap. We concluded that some of the fringes might be related to sound and other collective effects that occur when two condensates at fairly high density "touch" each other. I presented those results at the Sydney meeting only to illustrate we were able to do experiments with two condensates, but now we had to sort out what was happening.

It took us four more months until we observed clean interference between two condensates. When two condensates that were initially separated by a distance $d$ interfere and the interference pattern is recorded after a time $t$ of ballistic expansion, then the fringe spacing is the de Broglie wavelength $h / m v$ associated with the relative velocity $v=d / t$. For our geometry with two condensates about $100 \mu \mathrm{m}$ in length, we estimated that we would need at least $60 \mathrm{~ms}$ of time of flight to observe fringes with a $10-\mu \mathrm{m}$ period, close to the resolution of our imaging system. Unfortunately, due to gravity, the atoms dropped out of the field of view of our windows after $40 \mathrm{~ms}$. So we tried to gain a longer expansion time in a fountain geometry where we magnetically launched the atoms and observed them when they fell back through the observation region after more than $100 \mathrm{~ms}$ (Townsend et al., 1997), but the clouds were distorted. We also tried to compensate gravity by a vertical magnetic-field gradient. Some time later I learned about new calculations by the theory group at the Max Planck Institute in Garching, showing that the effective separation of two elongated condensates is smaller than their center-of-mass separation (Röhrl et al., 1997). This meant that we could observe interference fringes after only $40 \mathrm{~ms}$, just before the atoms fell out of the observation region. We immediately had a discussion in the group and decided to stop working on fountains and "antigravity" and simply let the atoms fall by $8 \mathrm{~mm}$ during $40 \mathrm{~ms}$.

We made some ambiguous observations where we saw low-contrast fringes together with some optical interference patterns of the probe light, but the breakthrough came on November 21, 1996, when we observed striking interference patterns (Fig. 15). I still remember the situation late that night when we wondered how could we prove beyond all doubt that these were matter-wave interference patterns and not some form of self-diffraction of a condensate confined by a light sheet and then released. We came up with the idea of eliminating one of the condensates in the last moment by focusing resonant yellow light on it. Whimsically, this laser beam was dubbed the "flame thrower." If the fringes were selfdiffraction due to the sharp edge in the confinement, they would remain; if they were true interference they would vanish. This was like a double slit experiment in 


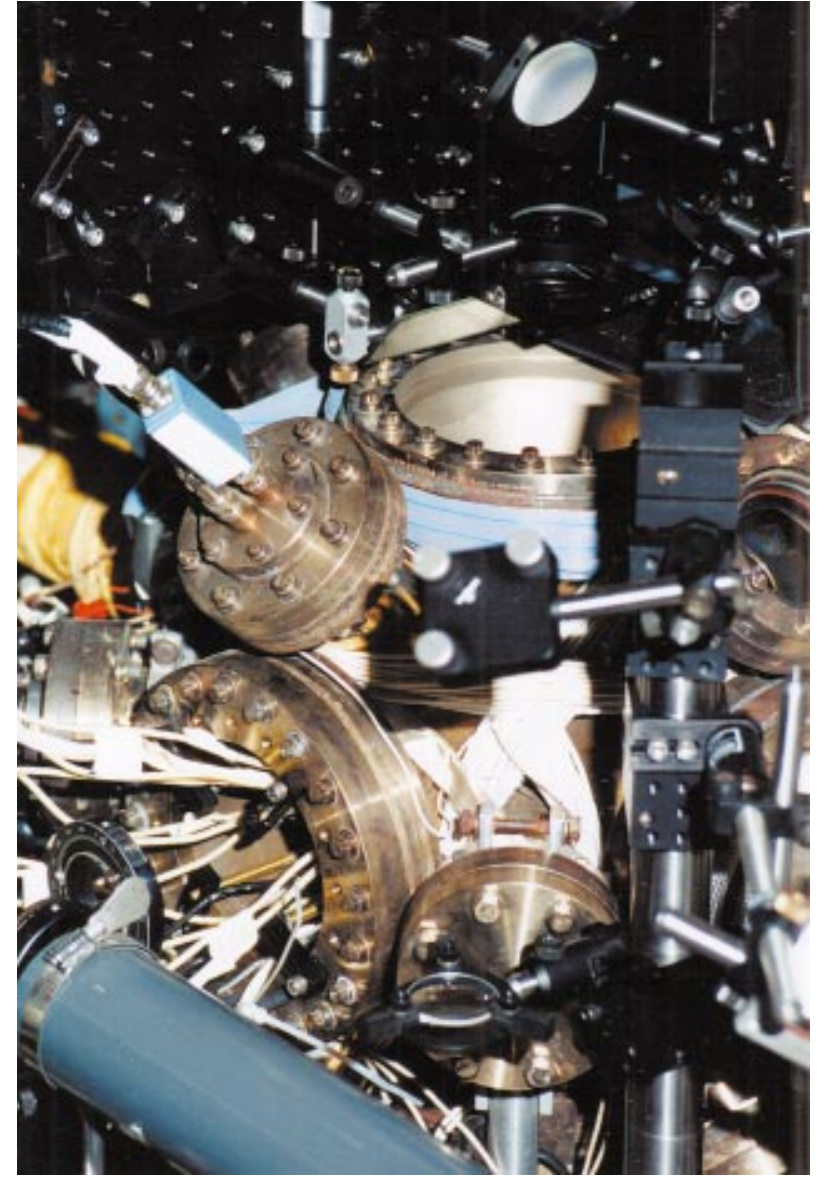

FIG. 12. Experimental setup for cooling sodium atoms to Bose-Einstein condensation around 1996. The atoms are trapped and cooled in the center of the ultrahigh-vacuum (UHV) chamber. The atomic beam oven and the Zeeman slower are to the left (outside the photo). The cloverleaf magnetic trap was mounted horizontally in reentrant flanges. Only the leads for the current and water cooling are visible. The diagonal flange above accommodated a BNC feedthrough for radio-frequency fields which were used to control the evaporative cooling. The lens and the mirror above the chamber were used to observe the condensate by dispersive or absorption imaging [Color].

optics where you cover one of the slits. It took a few hours to align the new laser beam, and we verified in phase-contrast imaging that we were able to selectively eliminate one of the two condensates.

We had a switch in our control panel which toggled between condensate elimination on and off. Then we went back and aligned the setup for the observation of interference. When we toggled the switch we had to wait for about half a minute until a new condensate was produced. This was the moment of truth. If the fringes appeared without a second condensate, then Nature would have fooled us for the whole night—but they disappeared and an enormous tension disappeared, as well. It was already early the next morning, with people arriving to work. I walked to Dan Kleppner's office and told him there was something he should see. So he shared the moment with us where we toggled the switch on alternating cooling cycles and correspondingly, the interfer-

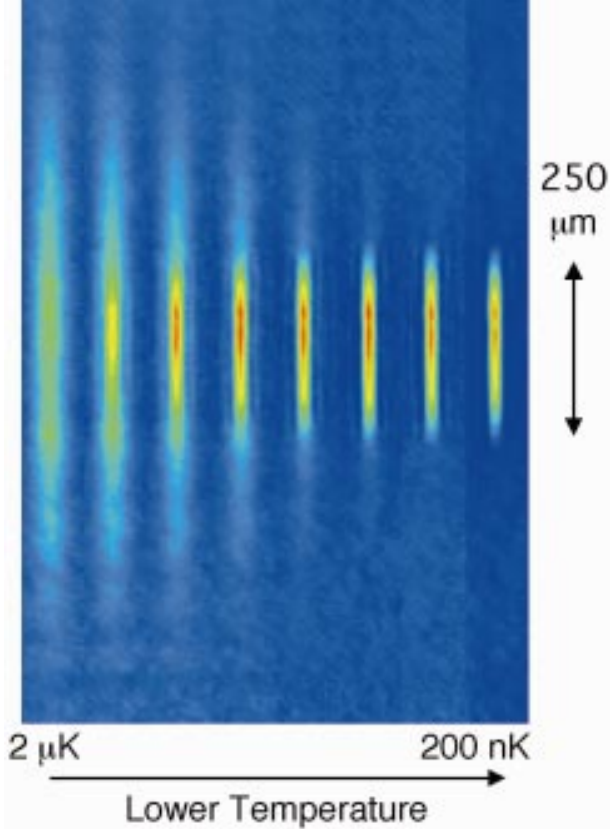

FIG. 13. Phase contrast images of trapped Bose gases across the BEC phase transition. At high temperature, above the $\mathrm{BEC}$ transition temperature, the density profile of the gas is smooth. As the temperature drops below the BEC phase transition, a high-density core of atoms appears in the center of the distribution. This is the Bose-Einstein condensate. Lowering the temperature further, the condensate number grows and the thermal wings of the distribution become shorter. Finally, the temperature drops to the point where a pure condensate with no discernible thermal fraction remains. Each image shows an equilibrated gas obtained in one complete trapping and cooling cycle. The axial and radial frequencies are about 17 and $230 \mathrm{~Hz}$, respectively.

ence pattern disappeared and reappeared. Interference between two light beams is quite a sight, but with atoms it is more dramatic. Destructive interference means that atoms plus atoms add up to vacuum!

The evidence for interference was so compelling that we submitted our paper based solely on the data of one experimental run (Andrews et al., 1997). This run is memorable to me for another reason: it was to be the last time I played a major role in preparing and running an experiment. During the night, I had put in the optics for the "flame thrower." Up to then, I was familiar with every piece of equipment in the lab and never thought this could change quickly, but it was like another phase transition. Hans-Joachim Miesner had just arrived, the first postdoc who stayed for more than a year, and he soon took over much responsibility for organizing the lab. There were more demands on my time to write papers and give talks, the group grew with the addition of two more graduate students (Shin Inouye and Chris Kuklewicz), and we had intensified our efforts to build a second BEC experiment. All this coincided in a few months. After earning my Ph.D. in 1986, I had spent eleven more years in the lab during three postdoc positions and as an assistant professor, but now began to play an advisory role. 


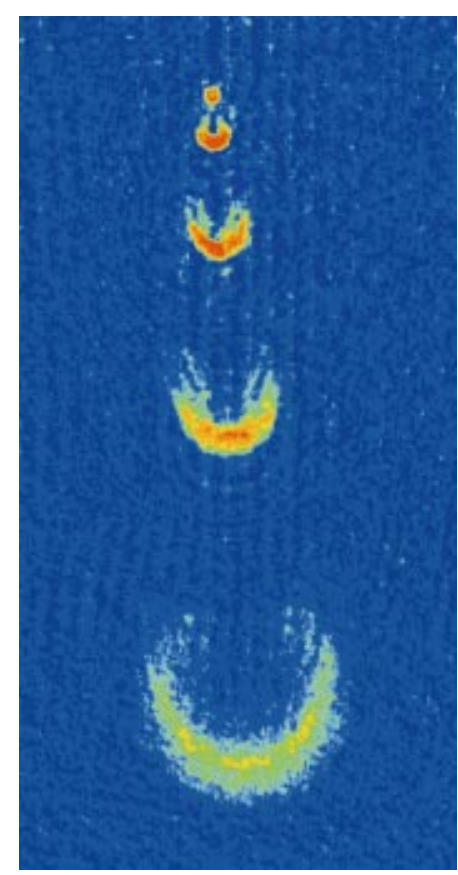

FIG. 14. The MIT atom laser operating at $200 \mathrm{~Hz}$. Pulses of coherent sodium atoms are coupled out from a Bose-Einstein condensate confined in a magnetic trap (field of view 2.5 $\times 5.0 \mathrm{~mm}^{2}$ ). Every $5 \mathrm{~ms}$, a short rf pulse transferred a fraction of these atoms into an unconfined quantum state. These atoms were accelerated downward by gravity and spread out due to repulsive interactions. The atom pulses were observed by absorption imaging. Each pulse contained between $10^{5}$ and $10^{6}$ atoms.

The papers on the rf output coupler (Mewes et al., 1997) and the interference (Andrews et al., 1997) of two condensates appeared in the same week in January 1997. Together they demonstrated the ability to create multiple pulses of coherent atoms, and have been regarded as the realization of an atom laser. The period starting with the early dreams of pursuing BEC and ending with the observation of the coherence of the condensate was remarkable. It was full of speculation, dreams, unknown physics, failures and successes, passion, excitement, and frustration. This period fused together a team of very different people who had one common denominator: the

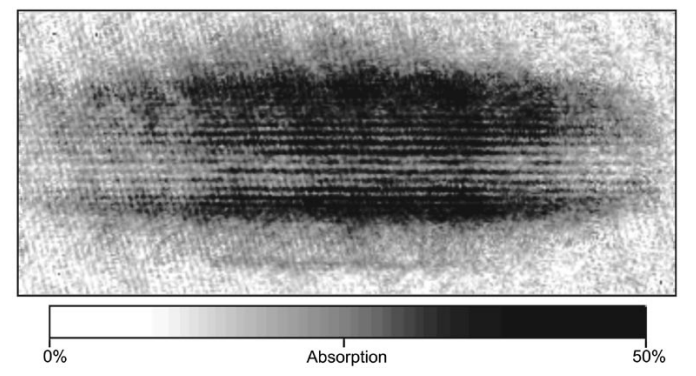

FIG. 15. Interference pattern of two expanding condensates observed after $40 \mathrm{~ms}$ time of flight. The width of the absorption image is $1.1 \mathrm{~mm}$. The interference fringes have a spacing of $15 \mu \mathrm{m}$ and are strong evidence for the long-range coherence of Bose-Einstein condensates.

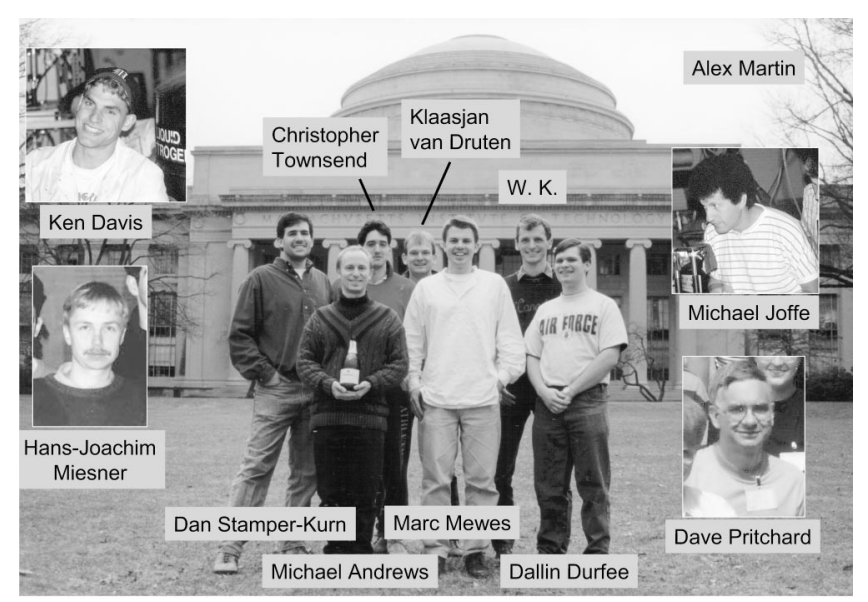

The team 1992 - 1996

FIG. 16. Team photo. This photo was taken in early 1996 in front of the MIT dome. The bottle of champagne was emptied to celebrate BEC in the cloverleaf trap. Names and photos of other collaborators during the period 1992-1996 have been added.

passion for experimental physics. It was a unique experience for me to work with these outstanding people (Fig. 16).

\section{THE MAGIC OF MATTER WAVES}

Many studies of BEC's have been performed over the last several years. The progress until 1998 is nicely summarized in the Varenna summer school proceedings (Inguscio et al., 1999). The studies that were most exciting for me displayed macroscopic quantum mechanics, the wavelike properties of matter on a macroscopic scale. These were also phenomena that no ordinary gas would show and illustrated dramatically that a new form of matter had been created. The interference of two condensates presented above (Fig. 15) is one such example. In the following, I want to discuss the amplification of atoms and the observation of lattices of quantized vortices.

These two examples are representative of the two areas into which research on gaseous BEC can be divided: in the first (which could be labeled "the atomic condensate as a coherent gas" or "atom lasers"), one would like to have as little interaction as possible-almost like the photons in a laser. The experiments are preferably done at low densities. The Bose-Einstein condensate serves as an intense source of ultracold coherent atoms for experiments in atom optics, in precision studies or for explorations of basic aspects of quantum mechanics. The second area could be labeled "BEC as a new quantum fluid" or "BEC as a many-body system." The focus here is on the interactions between the atoms that are most pronounced at high densities. The coherent amplification of atoms is an example of atom optics with condensates, and the study of vortices addresses the superfluid properties of the gas. 


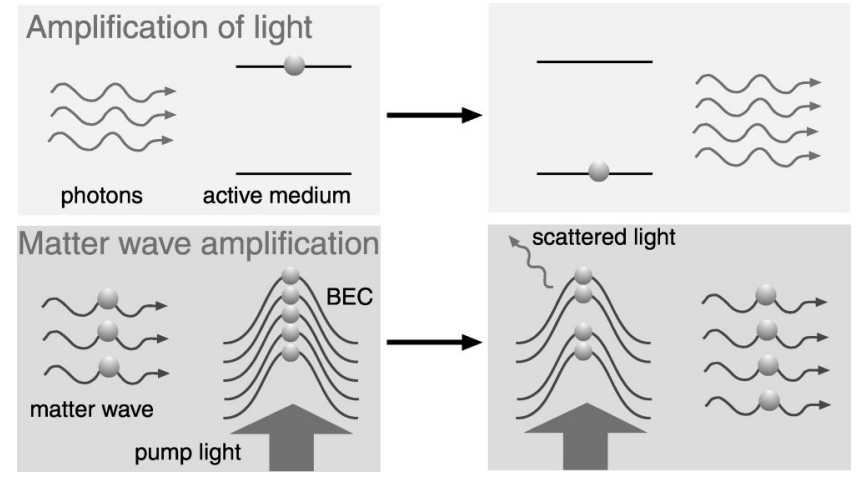

FIG. 17. Amplification of light and atoms: In the optical laser, light is amplified by passing it through an excited inverted medium. In the MIT atom amplifier, an input matter wave is sent through a Bose-Einstein condensate illuminated by laser light. Bosonic stimulation by the input atoms causes light to be scattered by the condensate exactly at the angle at which a recoiling condensate atom joins the input matter wave and augments it.

\section{A. Amplification of atoms in a Bose-Einstein condensate}

Since atoms are de Broglie waves, there are many analogies between atoms and light, which consists of electromagnetic waves. This is exploited in the field of atom optics where atoms are reflected, diffracted, and interfere using various atom-optical elements (Adams et al., 1994). One important question was whether these analogies can be extended to the optical laser, which is based on the amplification of light. When our group demonstrated a rudimentary atom laser in 1997 we had solved the problem of outcoupling (or extracting) atoms from the BEC and of verifying their coherence. The atomic amplification process happened during the formation of the Bose-Einstein condensate (Miesner et al., 1998) which is quite different from the way light is amplified in passing through an active medium. It was only in 1999 that our group managed to observe the amplification of atoms passing through another cloud of atoms serving as the active medium [Inouye, Pfau, et al., 1999 (simultaneously with the group in Tokyo; Kozuma et al., 1999)].

Amplifying atoms is more subtle than amplifying electromagnetic waves because atoms can only change their quantum state and cannot be created. Therefore, even if one could amplify gold atoms, one would not realize the dreams of medieval alchemy. An atom amplifier converts atoms from the active medium into an atomic wave that is exactly in the same quantum state as the input wave (Fig. 17).

The atom amplifier requires a reservoir, or an active medium, of ultracold atoms that have a very narrow spread of velocities and can be transferred to the atomic beam. A natural choice for the reservoir was a BoseEinstein condensate. One also needs a coupling mechanism that transfers atoms from the reservoir at rest to an input mode while conserving energy and momentum. This transfer of atoms was accomplished by scattering laser light. The recoil of the scattering process acceler-

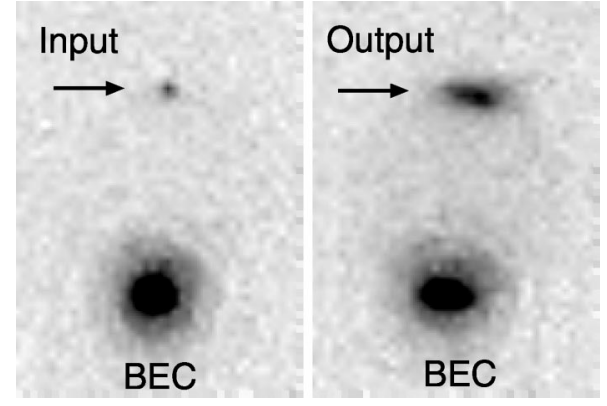

FIG. 18. Observation of atom amplification. Atom amplification is probed by sending an input beam through the atom amplifier, which is a Bose-Einstein condensate (BEC) illuminated with laser light. On the left side, the input beam has passed through the condensate without amplification. Some 20 ms later, a shadow picture is taken of the condensate and the input atoms. When the amplification process was activated by illuminating the condensate with laser light, the output pulse contained many more atoms than the input pulse-typical amplification factors were between 10 and 100 . The field of view is $1.9 \times 2.6 \mathrm{~mm}^{2}$.

ated some atoms to exactly match the velocity of the input atoms (Fig. 18). Not only were the atoms amplified, but they were in exactly the same motional state as the input atoms, i.e., they had the same quantummechanical phase. This was verified by interfering the amplified output with a copy of the input wave and observing phase coherence.

This direct observation of atom amplification in the summer of 1999 was preceded by a surprising occurrence late one night in October 1998 when we discovered a new form of superradiance (Inouye, Chikkatur, et al., 1999). We were studying Bragg spectroscopy (Stenger et al., 1999) and illuminated a BEC with two laser beams. I had no role in the running of the experiment and was working in my office, when around midnight the students came from the lab and told me that they saw atoms shooting out from the condensate with a velocity component perpendicular to the direction of the laser beams. We expected atoms to receive recoil momentum only along the laser beams, and all motion perpendicular to it to be diffuse due to the random direction of spontaneous Rayleigh scattering.

The whole lab started to discuss what was going on. With a running machine, everything could be tried out immediately. The first ideas were mundane: let's illuminate the condensate with only one laser beam and see what happens (the directional beams remained). We scrutinized the experimental setup for bouncing laser beams or beams which had not been completely switched off, but we found nothing. Increasingly, we considered that the observed phenomenon was genuine and not due to some experimental artifact. Knowing that the condensate was pencil shaped, the idea of laser emission along the long condensate axis came up, and this was already very close. We decided to stop the general discussion and continue taking data; the machine was running well and we wanted to take advantage of it. So some students, including Shin Inouye and Ananth Chikkatur, characterized the phenomenon, while Dan 


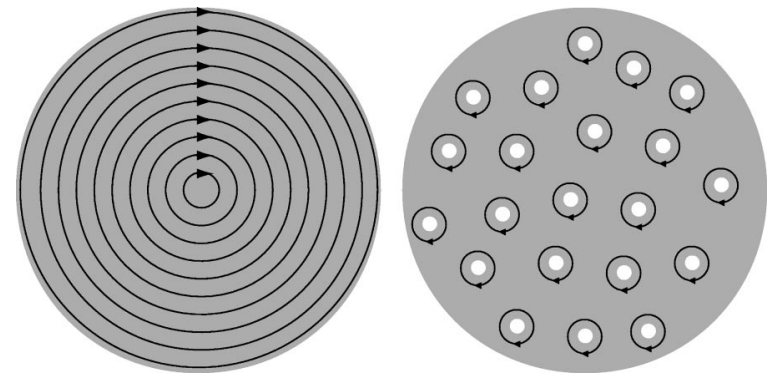

FIG. 19. Comparison of the flow fields of rotating normal liquids and superfluids. A normal fluid undergoes rigid body rotation, whereas a superfluid develops an array of quantized vortices.

Stamper-Kurn and I went to a blackboard and tried to figure out what was going on. Within the next hour, we developed the correct semiclassical description of superradiance in a condensate. In the lab, the predicted strong dependence on laser polarization was verified. A few months later we realized how we could use the superradiant amplification mechanism to build a phasecoherent atom amplifier. However, the labs were undergoing complete renovation at this point and we had to wait until the machine was running again before the phase-coherent amplification was implemented.

The demonstration of an atom amplifier added a new element to atom optics. In addition to passive elements like beam splitters, lenses, and mirrors, there is now an active atom-optical element. Coherent matter wave amplifiers may improve the performance of atom interferometers by making up for losses inside the device or by amplifying the output signal. Atom interferometers are already used as precise gravity and rotation sensors.

\section{B. Observation of vortex lattices in Bose-Einstein condensates}

Quantum mechanics and the wave nature of matter have subtle manifestations when particles have angular momentum, or more generally, when quantum systems are rotating. When a quantum-mechanical particle moves in a circle the circumference of the orbit has to be an integer multiple of the de Broglie wavelength. This quantization rule leads to the Bohr model and the discrete energy levels of the hydrogen atom. For a rotating superfluid, it leads to quantized vortices (Nozières and Pines, 1990). If one spins a normal liquid in a bucket, the fluid will finally rotate as a rigid body where the velocity smoothly increases from the center to the edge (Fig. 19, left). However, this smooth variation is impossible for particles in a single quantum state. To fulfill the abovementioned quantization rule, the flow field has to develop singular regions where the number of de Broglie wavelengths on a closed path jumps up by one. One possibility would be a radially symmetric flow field with concentric rings. Between adjacent rings, the number of de Broglie wavelengths on a circumference would change by one.

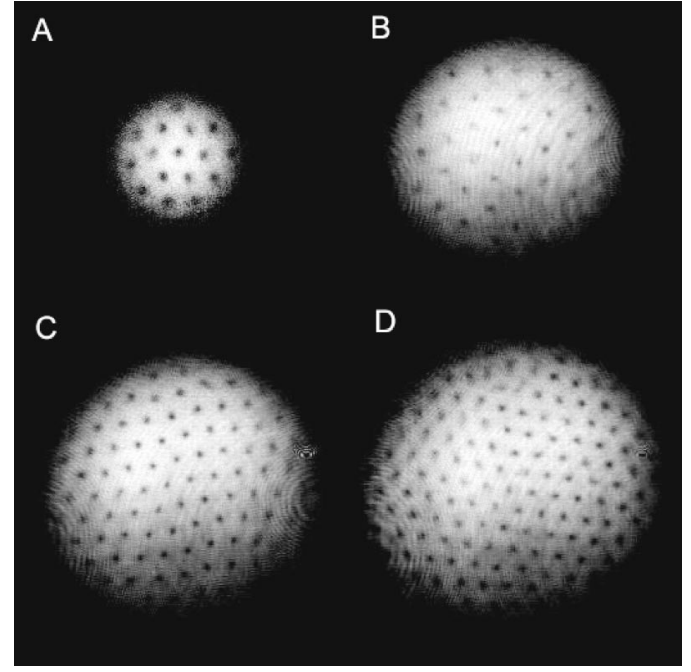

FIG. 20. Observation of vortex lattices in rotating BoseEinstein condensates. The examples shown contain (A) 16, (B) 32, (C) 80, and (D) 130 vortices as the speed of rotation was increased. The vortices have "crystallized" in a triangular pattern. The diameter of the cloud in (D) was $1 \mathrm{~mm}$ after ballistic expansion, which represents a magnification of 20. (Reprinted with permission from Abo-Shaeer et al., 2001. Copyright 2001 American Association for the Advancement of Science.)

However, the energetically most favorable configuration is achieved when the singularities in the velocity field are not distributed on cylindrical shells, but on lines. This corresponds to an array of vortices. In contrast to classical vortices like those in tornados or in a flushing toilet, the vortices in a Bose-Einstein condensate are quantized: when an atom goes around the vortex core, its quantum-mechanical phase changes by exactly $2 \pi$. Such quantized vortices play a key role in superfluidity and superconductivity. In superconductors, magnetic flux lines arrange themselves in regular lattices that have been directly imaged. In superfluids, previous direct observations of vortices had been limited to small arrays (up to 11 vortices), both in liquid ${ }^{4} \mathrm{He}$ (Yarmchuk et al., 1979) and in rotating gaseous Bose-Einstein condensates (BEC's) by a group in Paris (Madison et al., 2000).

In 2001, our group observed the formation of highlyordered vortex lattices in a rotating Bose-condensed gas (Abo-Shaeer et al., 2001). They were produced by spinning laser beams around the condensate, thus setting it into rotation. The condensate then exhibited a remarkable manifestation of quantum mechanics at a macroscopic level. The rotating gas cloud was riddled with more than 100 vortices. Since the vortex cores were smaller than the optical resolution, the gas was allowed to ballistically expand after the magnetic trap was switched off. This magnified the spatial structures 20fold. A shadow picture of these clouds showed little bright spots where the light penetrated through the empty vortex cores as if through tunnels (Fig. 20 shows a negative image).

A striking feature of the observed vortex lattices is the extreme regularity, free of any major distortions, even 

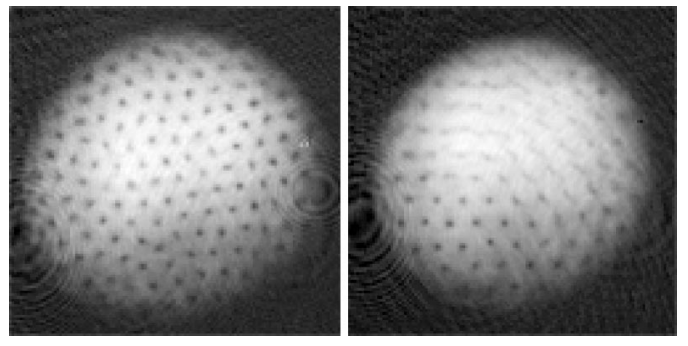

FIG. 21. Vortex lattices with defects. In the left image, the lattice has a dislocation near the center of the condensate. In the right one, there is a defect reminiscent of a grain boundary. (Reprinted with permission from Abo-Shaeer et al., 2001. Copyright 2001 American Association for the Advancement of Science.)

near the boundary. Such "Abrikosov" lattices were first predicted for quantized magnetic flux lines in type-II superconductors. However, Nature is not always perfect: some of the images showed distortions or defects of the vortex lattices; two examples are shown in Fig. 21. The physics of vortices is very rich. Subsequent work by my group and others has started to address the dynamics and nonequilibrium properties of vortex structures. How are vortices formed? How do they decay? Are the vortices straight or bent? Such experiments can be directly compared with first-principles calculations, which are possible for such a dilute system. This interplay between theory and experiment may lead to a better understanding of superfluidity and macroscopic quantum phenomena.

\section{OUTLOOK}

The rapid pace of developments in atomic BEC during the last few years has taken the community by surprise. After decades of searching for an elusive goal, nobody expected that condensates would be so robust and relatively easy to manipulate. Further, nobody imagined that such a simple system would pose so many challenges, not only to experimentalists, but also to our fundamental understanding of physics. The list of future challenges, both for theorists and for experimentalists, is long and includes the exploration of superfluidity and second sound in Bose gases, the physics of correlations and nonclassical wave functions (phenomena beyond the Gross-Pitaevskii equation), the study of quantumdegenerate molecules and Fermi gases, the development of practical "high-power" atom lasers, and their application in atom optics and precision measurements. These scientific goals are closely interwoven with technological advances to produce new single- or multi-species quantum-degenerate systems and novel ways of manipulation, e.g., using microtraps and atom chips. There is every indication for more excitement to come!

Work on BEC at MIT has been a tremendous team effort, and I am grateful to the past and present collaborators who have shared both the excitement and the hard work: J. R. Abo-Shaeer, M. R. Andrews, M. Boyd, G. Campbell, A. P. Chikkatur, J.-K. Chin, K. B. Davis,

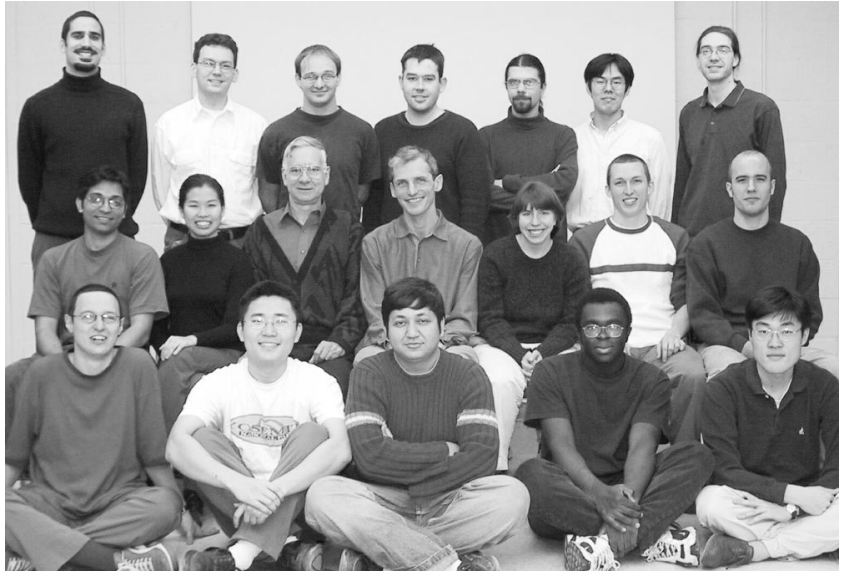

FIG. 22. The author with his team in November 2001. Front row, from left to right: Z. Hadzibabic, K. Xu, S. Gupta, E. Tsikata, Y.-I. Shin. Middle row: A.P. Chikkatur, J.-K. Chin, D.E. Pritchard, W. K., G. Campbell, A.E. Leanhardt, M. Boyd. Back row: J.R. Abo-Shaeer, D. Schneble, J.M. Vogels, K. Dieckmann, C.A. Stan, Y. Torii, E. Streed.

K. Dieckmann, D. S. Durfee, A. Görlitz, S. Gupta, T. L. Gustavson, Z. Hadzibabic, S. Inouye, M. A. Joffe, D. Kielpinski, M. Köhl, C. E. Kuklewicz, A. E. Leanhardt, R. F. Löw, A. Martin, M.-O. Mewes, H.-J. Miesner, R. Onofrio, T. Pfau, D. E. Pritchard, C. Raman, D. Schneble, C. Schunck, Y.-I. Shin, D. M. Stamper-Kurn, C. A. Stan, J. Stenger, E. Streed, Y. Torii, C. G. Townsend, N. J. van Druten, J. M. Vogels, K. Xu, M. W. Zwierlein, and many MIT undergraduate students. Exemplary administrative support has been provided by Carol Costa for more than 12 years. Figure 22 shows the team in November 2001. Special thanks go to Dan Kleppner and Tom Greytak for inspiration and constant encouragement. The author also acknowledges the fruitful interactions with colleagues all over the world who

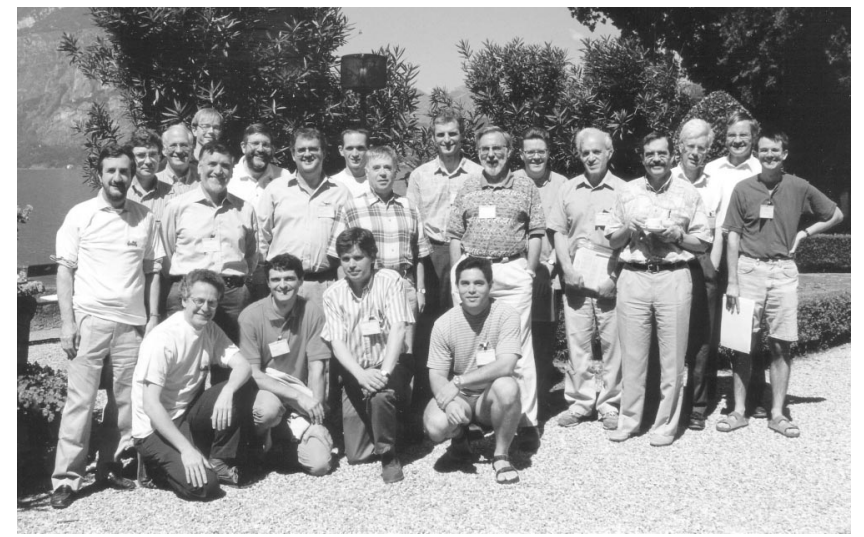

FIG. 23. Lecturers, seminar speakers, and directors at the summer school on "Bose-Einstein Condensation in Atomic Gases" in Varenna, July 7-17, 1998. Front row: Jean Dalibard, Guglielmo Tino, Fernando Sols, Kris Helmerson. Back row: Sandro Stringari, Carl Wieman, Alexander Fetter, Tilman Esslinger, Massimo Inguscio, William Phillips, Daniel Heinzen, Peter Fedichev, Lev Pitaevskii, W. K., Allan Griffin, Keith Burnett, Daniel Kleppner, Alain Aspect, Ennio Arimondo, Theodor Hänsch, Eric Cornell. 
have contributed to this rich and exciting field. Some of these colleagues are depicted in Fig. 23, which is a group photo of the lecturers at the Varenna summer school on BEC in 1998. In particular, the yearlong competition with the group at Boulder led by Eric Cornell and Carl Wieman inspired the best from me and my team, and despite tight competition, there has been genuine collegiality and friendship. I want to thank the Office of $\mathrm{Na}$ val Research, the National Science Foundation, the Army Research Office, the Joint Services Electronics Program, NASA, and the David and Lucile Packard Foundation for their encouragement and financial support of this work.

\section{REFERENCES}

Abo-Shaeer, J. R., C. Raman, J. M. Vogels, and W. Ketterle, 2001, Science 292, 476.

Adams, C. S., and E. Riis, 1997, Prog. Quantum Electron. 21, 1 .

Adams, C. S., M. Sigel, and J. Mlynek, 1994, Phys. Rep. 240, 143.

Anderson, M. H., J. R. Ensher, M. R. Matthews, C. E. Wieman, and E. A. Cornell, 1995, Science 269, 198.

Andrews, M. R., M.-O. Mewes, N. J. van Druten, D. S. Durfee, D. M. Kurn, and W. Ketterle, 1996, Science 273, 84.

Andrews, M. R., C. G. Townsend, H.-J. Miesner, D. S. Durfee, D. M. Kurn, and W. Ketterle, 1997, Science 275, 637.

Arimondo, E., W. D. Phillips, and F. Strumia, 1992, Eds., Laser Manipulation of Atoms and Ions, Proceedings of the International School of Physics Enrico Fermi, Course CXVIII (North-Holland, Amsterdam).

Bagnato, V. S., G. P. Lafyatis, A. G. Martin, E. L. Raab, R. N. Ahmad-Bitar, and D. E. Pritchard, 1987, Phys. Rev. Lett. 58, 2194.

Barrett, T. E., S. W. Dapore-Schwartz, M. D. Ray, and G. P. Lafyatis, 1991, Phys. Rev. Lett. 67, 3483.

Baumberg, J. J., 2002, Phys. World March, 37.

Bose, S. N., 1924, Z. Phys. 26, 178.

Bradley, C. C., C. A. Sackett, J. J. Tollet, and R. G. Hulet, 1995, Phys. Rev. Lett. 75, 1687.

Bradley, C. C., C. A. Sackett, J. J. Tollet, and R. G. Hulet, 1997, Phys. Rev. Lett. 79, 1170.

Butov, L. V., C. W. Lai, A. L. Ivanov, A. C. Gossard, and D. S. Chemla, 2002, Nature (London) 417, 47.

Cho, H. and G. A. Williams, 1995, Phys. Rev. Lett. 75, 1562.

Chu, S., 1998, Rev. Mod. Phys. 70, 685.

Chu, S., L. Hollberg, J. E. Bjorkholm, A. Cable, and A. Ashkin, 1985, Phys. Rev. Lett. 55, 48.

Cline, R. W., D. A. Smith, T. J. Greytak, and D. Kleppner, 1980, Phys. Rev. Lett. 45, 2117.

Cohen-Tannoudji, C. N., 1998, Rev. Mod. Phys. 70, 707.

Cornell, E. A., C. Monroe, and C. E. Wieman, 1991, Phys. Rev. Lett. 67, 2439.

Crooker, B. C., B. Hebral, E. N. Smith, Y. Takano, and J. D. Reppy, 1983, Phys. Rev. Lett. 51, 666.

Davis, K. B., M.-O. Mewes, M. R. Andrews, N. J. van Druten, D. S. Durfee, D. M. Kurn, and W. Ketterle, 1995, Phys. Rev. Lett. 75, 3969.

Davis, K. B., M.-O. Mewes, M. A. Joffe, M. R. Andrews, and W. Ketterle, 1995, Phys. Rev. Lett. 74, 5202.
Davis, K. B., M. O. Mewes, M. A. Joffe, and W. Ketterle, 1994, in Fourteenth International Conference on Atomic Physics, Boulder, Colorado, 1994, Book of Abstracts, 1-M3 (University of Colorado, Boulder, Colorado).

DePue, M. T., C. McCormick, S. L. Winoto, S. Oliver, and D. S. Weiss, 1999, Phys. Rev. Lett. 82, 2262.

Doyle, J. M., J. C. Sandberg, I. A. Yu, C. L. Cesar, D. Kleppner, and T. J. Greytak, 1991, Phys. Rev. Lett. 67, 603.

Drewsen, M., P. Laurent, A. Nadir, G. Santarelli, A. Clairon, Y. Castin, D. Grison, and C. Salomon, 1994, Appl. Phys. B: Lasers Opt. B59, 283.

Durfee, D. S., and W. Ketterle, 1998, Opt. Express 2, 299.

Einstein, A., 1925a, Sitzungsber. Preuss. Akad. Wiss., Phys. Math. Kl. Bericht 1, 3.

Einstein, A., 1925b, Sitzungsber. Preuss. Akad. Wiss., Phys. Math. Kl. Bericht 3, 18.

Fortin, E., E. Benson, and A. Mysyrowicz, 1995, in BoseEinstein Condensation, edited by A. Griffin, D. W. Snoke, and S. Stringari (Cambridge University Press, Cambridge) pp. 519-523.

Fried, D. G., T. C. Killian, L. Willmann, D. Landhuis, S. C. Moss, D. Kleppner, and T. J. Greytak, 1998, Phys. Rev. Lett. 81, 3811.

Gavroglu, K., 1995, Fritz London: A Scientific Biography (Cambridge University Press, Cambridge).

Goldman, V. V., I. F. Silvera, and A. J. Leggett, 1981, Phys. Rev. B 24, 2870.

Greytak, T. J., 1995, in Bose-Einstein Condensation, edited by A. Griffin, D. W. Snoke, and S. Stringari (Cambridge University Press, Cambridge), pp. 131-159.

Greytak, T. J., and D. Kleppner, 1984, in New Trends in Atomic Physics, Les Houches Summer School 1982, edited by G. Grynberg and R. Stora (North-Holland, Amsterdam), p. 1125.

Griffin, A., 1999, in Bose-Einstein Condensation in Atomic Gases, Proceedings of the International School of Physics Enrico Fermi, Course CXL, edited by M. Inguscio, S. Stringari, and C. E. Wieman (IOS Press, Amsterdam), p. 1.

Griffin, A., D. W. Snoke, and S. Stringari, 1995, Eds., BoseEinstein Condensation (Cambridge University Press, Cambridge).

Hecht, C. E., 1959, Physica (Amsterdam) 25, 1159.

Heinzen, D. J., 1999, in Bose-Einstein Condensation in Atomic Gases, Proceedings of the International School of Physics Enrico Fermi, Course CXL, edited by M. Inguscio, S. Stringari, and C. E. Wieman (IOS, Amsterdam), pp. 351-390.

Helmerson, K., A. Martin, and D. E. Pritchard, 1992, J. Opt. Soc. Am. B 9, 1988.

Hess, H. F., 1986, Phys. Rev. B 34, 3476.

Huang, K., 1987, Statistical Mechanics (Wiley, New York).

Huse, D. A., and E. Siggia, 1982, J. Low Temp. Phys. 46, 137. Ido, T., Y. Isoya, and H. Katori, 2000, Phys. Rev. A 61, 061403. Inguscio, M., S. Stringari, and C. E. Wieman, 1999, Eds., BoseEinstein Condensation in Atomic Gases, Proceedings of the International School of Physics Enrico Fermi, Course CXL (IOS, Amsterdam).

Inouye, S., A. P. Chikkatur, D. M. Stamper-Kurn, J. Stenger, D. E. Pritchard, and W. Ketterle, 1999, Science 285, 571.

Inouye, S., T. Pfau, S. Gupta, A. P. Chikkatur, A. Görlitz, D. E. Pritchard, and W. Ketterle, 1999, Nature (London) 402, 641. Joffe, M. A., W. Ketterle, A. Martin, and D. E. Pritchard, 1993, J. Opt. Soc. Am. B 10, 2257.

Kasevich, M., and S. Chu, 1992, Phys. Rev. Lett. 69, 1741. 
Kerman, A. J., V. Vuletic, C. Chin, and S. Chu, 2000, Phys. Rev. Lett. 84, 439.

Ketterle, W., K. B. Davis, M. A. Joffe, A. Martin, and D. E. Pritchard, 1993a, Phys. Rev. Lett. 70, 2253.

Ketterle, W., K. B. Davis, M. A. Joffe, A. Martin, and D. E. Pritchard, 1993b, talk given at OSA Annual Meeting, Toronto, Canada, October 3-8.

Ketterle, W., D. S. Durfee, and D. M. Stamper-Kurn, 1999, in Bose-Einstein Condensation in Atomic Gases, Proceedings of the International School of Physics Enrico Fermi, Course $C X L$, edited by M. Inguscio, S. Stringari, and C. E. Wieman (IOS Press, Amsterdam), pp. 67-176.

Ketterle, W., and S. Inouye, 2001, C. R. Acad. Sci., Ser IV: Phys., Astrophys. 2, 339.

Ketterle, W., A. Martin, M. A. Joffe, and D. E. Pritchard, 1992, Phys. Rev. Lett. 69, 2483.

Ketterle, W., and D. E. Pritchard, 1992a, Appl. Phys. B: Photophys. Laser Chem. B54, 403.

Ketterle, W., and D. E. Pritchard, 1992b, Phys. Rev. A 46, 4051.

Ketterle, W., and J. J. van Druten, 1996, in Advances in Atomic, Molecular, and Optical Physics, edited by B. Bederson and H. Walther (Academic, San Diego), Vol. 37, pp. 181236.

Kleppner, D., T. J. Greytak, T. C. Killian, D. G. Fried, L. Willmann, D. Landhuis, and S. C. Moss, 1999, in Bose-Einstein Condensation in Atomic Gases, Proceedings of the International School of Physics Enrico Fermi, Course CXL, edited by M. Inguscio, S. Stringari, and C. E. Wieman (IOS Press, Amsterdam), pp. 177-1999.

Kozuma, M., Y. Suzuki, Y. Torii, T. Sugiura, T. Kuga, E. W. Hagley, and L. Deng, 1999, Science 286, 2309.

Letokhov, V. S., and V. G. Minogin, 1980, Opt. Commun. 35, 199.

Lett, P. D., W. D. Phillips, S. L. Rolston, C. E. Tanner, R. N. Watts, and C. I. Westbrook, 1989, J. Opt. Soc. Am. B 6, 2084. Lin, J. L., and J. P. Wolfe, 1993, Phys. Rev. Lett. 71, 1222.

Lindquist, K., M. Stephens, and C. Wieman, 1992, Phys. Rev. A 46, 4082.

Lovelace, R. V. E., C. Mahanian, T. J. Tommila, and D. M. Lee, 1985, Nature (London) 318, 30.

Madison, K. W., F. Chevy, W. Wohlleben, and J. Dalibard, 2000, J. Mod. Opt. 47, 2715.

Masuhara, N., J. M. Doyle, J. C. Sandbert, D. Kleppner, T. J. Greytak, H. F. Hess, and G. P. Kochanski, 1988, Phys. Rev. Lett. 61, 935.

Metcalf, H., and P. van der Straten, 1994, Phys. Rep. 244, 203. Mewes, M.-O., M. R. Andrews, D. M. Kurn, D. S. Durfee, C. G. Townsend, and W. Ketterle, 1997, Phys. Rev. Lett. 78, 582. Mewes, M.-O., M. R. Andrews, N. J. van Druten, D. M. Kurn, D. S. Durfee, and W. Ketterle, 1996a, Phys. Rev. Lett. 77, 416. Mewes, M.-O., M. R. Andrews, N. J. van Druten, D. M. Kurn, D. S. Durfee, C. G. Townsend, and W. Ketterle, 1996b, Phys. Rev. Lett. 77, 988.

Miesner, H.-J., D. M. Stamper-Kurn, M. R. Andrews, D. S. Durfee, S. Inouye, and W. Ketterle, 1998, Science 279, 1005. Migdall, A. L., J. V. Prodan, W. D. Phillips, T. H. Bergeman, and H. J. Metcalf, 1985, Phys. Rev. Lett. 54, 2596.

Monroe, C., E. Cornell, and C. Wieman, 1992, in Laser Manipulation of Atoms and Ions, Proceedings of the International School of Physics Enrico Fermi, Course CXVIII, edited by E. Arimondo, W. D. Phillips, and F. Strumia (NorthHolland, Amsterdam), pp. 361-377.
Monroe, C., W. Swann, H. Robinson, and C. Wieman, 1990, Phys. Rev. Lett. 65, 1571.

Nozières, P., and D. Pines, 1990, The Theory of Quantum Liquids (Addison-Wesley, Redwood City, CA).

O’Hara, K. E., L. Ó. Súilleabháin, and J. P. Wolfe, 1999, Phys. Rev. B 60, 10565.

Oliva, J., 1989, Phys. Rev. B 39, 4197.

Pais, A., 1982, Subtle is the Lord, The Science and the Life of Albert Einstein (Clarendon, Oxford).

Petrich, W., M. H. Anderson, J. R. Ensher, and E. A. Cornell, 1994, J. Opt. Soc. Am. B 11, 1332.

Petrich, W., M. H. Anderson, J. R. Ensher, and E. A. Cornell, 1995, Phys. Rev. Lett. 74, 3352.

Phillips, W. D., 1998, Rev. Mod. Phys. 70, 721.

Pritchard, D. E., 1986, in Electronic and Atomic Collisions, Invited Papers of the XIV International Conference on the Physics of Electronic and Atomic Collisions, Palo Alto, California, July, 1985, edited by D. C. Lorents, W. E. Meyerhof, and J. R. Peterson (Elsevier, New York), pp. 593-604.

Pritchard, D. E., K. Helmerson, and A. G. Martin, 1989, in Atomic Physics 11, edited by S. Haroche, J. C. Gay, and G. Grynberg (World Scientific, Singapore), p. 179.

Pritchard, D. E., and W. Ketterle, 1992, in Laser Manipulation of Atoms and Ions, Proceedings of the International School of Physics Enrico Fermi, Course CXVIII, edited by E. Arimondo, W. D. Phillips, and F. Strumia (North-Holland, Amsterdam), pp. 473-496.

Rasolt, M., M. H. Stephen, M. E. Fisher, and P. B. Weichman, 1984, Phys. Rev. Lett. 53, 798.

Reppy, J. D., 1984, Physica B 126, 335.

Röhrl, A., M. Naraschewski, A. Schenzle, and H. Wallis, 1997, Phys. Rev. Lett. 78, 4143.

Saba, M., C. Ciuti, J. Bloch, V. Thierry-Mieg, R. André, L. S. Dang, S. Kundermann, A. Mura, G. Bongiovanni, J. Sl. Staehli, and B. Deveaud, 2001, Nature (London) 414, 731.

Safonov, A. I., S. A. Vasilyev, L. S. Yasnikov, I. I. Lukashevich, and S. Jaakola, 1998, Phys. Rev. Lett. 81, 4545.

Schrödinger, E., 1952, Statistical Thermodynamics (Cambridge University Press, Cambridge); reprinted by Dover Publications (New York, 1989).

Silvera, I. F., and J. T. M. Walraven, 1980, Phys. Rev. Lett. 44, 164.

Silvera, I. F., and J. T. M. Walraven, 1986, in Progress in Low Temperature Physics, edited by D. F. Brewer (Elsevier, Amsterdam) Vol. X, p. 139.

Stamper-Kurn, D., and W. Ketterle, 2001, in Coherent Atomic Matter Waves, Proceedings of the Les Houches Summer School, Course LXXII, 1999, edited by R. Kaiser, C. Westbrook, and F. David (Springer, New York).

Stenger, J., S. Inouye, A. P. Chikkatur, D. M. Stamper-Kurn, D. E. Pritchard, and W. Ketterle, 1999, Phys. Rev. Lett. 82, 4569.

Stoof, H. T. C., J. M. V. A. Koelman, and B. J. Verhaar, 1988, Phys. Rev. B 38, 4688.

Stwalley, W. C., and L. H. Nosanow, 1976, Phys. Rev. Lett. 36, 910.

Surkov, E. L., J. T. M. Walraven, and G. V. Shlyapnikov, 1996, Phys. Rev. A 53, 3403.

Taubes, G., 1994, Science 265, 184.

Townsend, C. G., N. H. Edwards, C. J. Cooper, K. P. Zetie, C. J. Foot, A. M. Steane, P. Szriftgiser, H. Perrin, and J. Dalibard, 1995, Phys. Rev. A 52, 1423.

Townsend, C. G., N. H. Edwards, K. P. Zetie, C. J. Cooper, J. Rink, and C. J. Foot, 1996, Phys. Rev. A 53, 1702. 
Townsend, C. G., N. J. van Druten, M. R. Andrews, D. S. Durfee, D. M. Kurn, M.-O. Mewes, and W. Ketterle, 1997, in Atomic Physics 15, Fifteenth International Conference on Atomic Physics, Amsterdam, August 1996, edited by H. B. van Linden van den Heuvell, J. T. M. Walraven, and M. W. Reynolds (World Scientific, Singapore), pp. 192-211.

Vigué, J., 1986, Phys. Rev. A 34, 4476.

Walker, T., and P. Feng, 1994, in Advances in Atomic, Molecular, and Optical Physics, edited by B. Bederson and $\mathrm{H}$. Walther (Academic, San Diego), Vol. 34, pp. 125-170.

Walker, T., D. Sesko, and C. Wieman, 1990, Phys. Rev. Lett. 64, 408.

Walraven, J. T. M., 1996, in Quantum Dynamics of Simple Sys- tems, edited by G. L. Oppo, S. M. Barnett, E. Riis, and M. Wilkinson (Institute of Physics, London), pp. 315-352.

Weiner, J., 1995, in Advances in Atomic, Molecular, and Optical Physics, edited by B. Bederson and H. Walther (Academic, San Diego), Vol. 35, pp. 45-78.

Weiner, J., V. S. Bagnato, S. Zilio, and P. S. Julienne, 1999, Rev. Mod. Phys. 71, 1.

Wolfe, J. P., J. L. Lin, and D. W. Snoke, 1995, in Bose-Einstein Condensation, edited by A. Griffin, D. W. Snoke, and S. Stringari (Cambridge University Press, Cambridge), pp. 281329.

Yamamoto, Y., 2000, Nature (London) 405, 629.

Yarmchuk, E. J., M. J. V. Gordon, and R. E. Packard, 1979, Phys. Rev. Lett. 43, 214. 